\title{
Revisión del género Polygonum (Polygonaceae) en Chile
}

\section{Polygonum (Polygonaceae) in Chile}

\section{Sebastián Teillier ${ }^{1, *}$, Raúl Peña ${ }^{2}$ \& Jorge Macaya-Berti ${ }^{3}$}

${ }^{1}$ Escuela de Arquitectura y Paisaje. Universidad Central de Chile, Santiago, Chile.

${ }^{2}$ Consultor independiente. San Francisco de Mostazal, Chile.

${ }^{3}$ Cedrem Consultores Ltda., Santiago, Chile.

*E-mail: steillier@gmail.com

\section{RESUMEN}

Polygonum L. sensu lato es un género de las poligonáceas del que se han descrito unas 200 especies herbáceas o subleñosas, distribuidas en todas las regiones templadas y cálidas del mundo. Dado que el género en Chile no había sido objeto de una revisión desde la de la flora de Gay, ésta se llevó a cabo en el marco del proyecto "Nueva Flora de Chile". Como resultado de la revisión se obtuvo que en Chile crecen once especies de Polygonum; dos posiblemente endémicas, P. bowenkampii Phil. y P. delfinii Phil.; dos nativas, $P$. hydropiperoides Michx. y P. maritimum L., siete alóctonas asilvestradas, $P$. acuminatum Kunth, P. aviculare L., P. campanulatum Hook. f., P. hydropiper L., P. lapathifolium L., P. orientale L. y P. persicaria L. Se designan los siguientes lectotipos: Polygonum araucanum Phil., Polygonum caballeroi Phil., Polygonum glareosum Phil., Polygonum sanguinaria, J. Remy, Polygonum valdivianum Phil. y Polygonum virgatum Cham. \& Schltdl.

Palabras clave: Nueva Flora de Chile, Polygonaceae.

\section{ABSTRACT}

Polygonum L. s.l. (Polygonaceae) included about 200 herbaceous or scrubby species, distributed in all temperate and warm regions of the world. Since the genus in Chile has not been reviewed since Claudio Gay's flora, the genus has been reviewed in the framework of the "New Flora of Chile" project. As a result of the taxonomic revision we establish that Polygonum in Chile is represented by eleven species; two possibly endemic, P. bowenkampii Phil. and P. delfinii Phil.; two native, P. hydropiperoides Michx. and P. maritimum L.; and seven alien, P. acuminatum Kunth, P. aviculare L., P. campanulatum Hook. f., P. hydropiper L., P. lapathifolium L., P. orientale L. and P. persicaria L. The following lectotypes are here designated: Polygonum araucanum Phil., Polygonum caballeroi Phil., Polygonum glareosum Phil., Polygonum sanguinaria, J. Remy, Polygonum valdivianum Phil., and Polygonum virgatum Cham. \& Schltdl.

Keywords: Nueva Flora de Chile, Polygonaceae.

\section{INTRODUCCIÓN}

Polygonum L. pertenece a las Polygonaceae y reúne al menos unas 150 especies herbáceas o subarbustivas en el mundo (Mabberley 1987, Li et al. 2003). Los límites del género han sido cuestionados en varios trabajos filogenéticos recientes tales como los de Ronse Decraene \& Akeroyd (1988), Choudhary et al. (2012) y Schuster et al. (2015), los que han asignado a las especies de Polygonum a géneros tales como Aconogonon (Meisn.) Rchb., Fallopia Lour., Koenigia L. o Persicaria Mill. En este tratamiento se tratan como Polygonum s.l. a las especies transferidas actualmente a Persicaria y Aconogonon; pero no se incluyen en él a aquellas incluidas en Koenigia o en Fallopia. En relación con la existencia de Polygonum en Chile, en los 
catálogos más recientes figuran desde once hasta catorce especies (Tabla 1), ocho de ellas han sido reportadas en los tres catálogos, en tanto que tres del catálogo de Marticorena \& Quezada (1985) no han vuelto a ser citadas, y cuatro o cinco especies han sido incluidas en los catálogos, tanto de Zuloaga et al. (2008) como de Rodríguez et al. (2018).

De acuerdo con los datos de la bibliografía ya referida, las especies de Polygonum se distribuyen geográficamente entre las regiones de Arica-Parinacota y de Magallanes, y están representadas también en el territorio insular, es decir, en la isla de Pascua (Matthei 1995) y en el archipiélago Juan Fernández (Fuentes et al. 2014). Respecto del hábitat, las especies de Polygonum subg. Polygonum son ruderales, andinas o litorales, en tanto que las de Polygonum subg. Persicaria (L.) Raf. son con frecuencia malezas agrícolas o plantas higrófilas que crecen a orillas de los canales, esteros y en otros tipos de humedales (Matthei 1995).

A la vista de los antecedentes y considerando que la última revisión del género al nivel de Chile fue propuesta por C. Gay (Rémy 1851), se plantea el objetivo de estudiar el género Polygonum con el fin de establecer cuáles son las especies que crecen en Chile y su actual distribución geográfica.

\section{MATERIALES Y MÉTODOS}

Para esta revisión se utilizó la metodología clásica, comparativa, de la taxonomía. Se revisó la bibliografía regional referente a Polygonum s.l. y algunos textos europeos, tanto en formato físico como digital, incluyendo las descripciones de las especies provistas por J. Rémy (en Gay 1851), por R.A. Philippi (1864, 1873 y 1895) y L.E. Navas (1976).

Se examinaron los ejemplares tipo físicos depositados en el herbario del Museo Nacional de Historia Natural (SGO) y, en forma digital, las fotos disponibles en el sitio JSTOR-Global Plants (https://about.jstor.org) y en los de los herbarios P, K, G, GH, NY, BM. Se revisaron especímenes de los herbarios CONC, SGO, SQF, VALP, parte de los que se citan como materiales estudiados (acrónimos de los herbarios según Thiers 2020, continuamente actualizado).

Con el fin de confirmar los nombres y establecer sinonimias taxonómicas o nomenclaturales, se consultaron las bases de datos Tropicos (https://www.tropicos.org), JSTOR (https:// www.plants.jstor.org) y la página web del sitio The Plant List (http://www.theplantlist.org). Finalmente, para establecer la distribución geográfica de los taxones se consultó la base de datos de la Flora del Cono Sur (Zuloaga et al. 2008) y el catálogo de Rodríguez et al. (2018).

TABLA 1. Polygonum en Chile. Reporte de las especies del género en Chile en los catálogos modernos. / Polygonum in Chile, species cited at modern catalogues.

\begin{tabular}{|c|c|c|c|c|}
\hline Especie/Publicación & $\begin{array}{l}\text { Marticorena \& } \\
\text { Quezada, } 1985\end{array}$ & $\begin{array}{l}\text { Zuloaga et al. } \\
2008\end{array}$ & $\begin{array}{l}\text { Rodríguez et } \\
\text { al., } 2018\end{array}$ & Esta publicación \\
\hline Polygonum acuminatum Kunth & & * & $*$ & $*$ \\
\hline Polygonum arenastrum Boreau & $*$ & & & \\
\hline Polygonum aviculare L. & * & * & $*$ & * \\
\hline Polygonum berteroi Phil. & * & & & \\
\hline Polygonum bowenkampii Phil. & & * & * & * \\
\hline Polygonum brasiliense K. Koch & & $*$ & * & \\
\hline Polygonum campanulatum Hook. f. & & * & $*$ & * \\
\hline Polygonum chiloensis Rech. F. & * & & & \\
\hline Polygonum delfinii Phil. & * & & & * \\
\hline Polygonum hydropiper L. & & * & * & * \\
\hline Polygonum hydropiperoides Michx. & * & * & * & * \\
\hline Polygonum lapathifolium L. & $*$ & $*$ & $*$ & * \\
\hline Polygonum maritimum L. & * & * & * & * \\
\hline Polygonum mite Schrank & * & * & * & \\
\hline Polygonum orientale L. & * & $*$ & * & * \\
\hline Polygonum persicaria L. & $*$ & * & * & * \\
\hline Polygonum sanguinaria J. Remy & * & * & * & \\
\hline Polygonum stypticum Cham. \& Schltdl. & & * & & \\
\hline
\end{tabular}




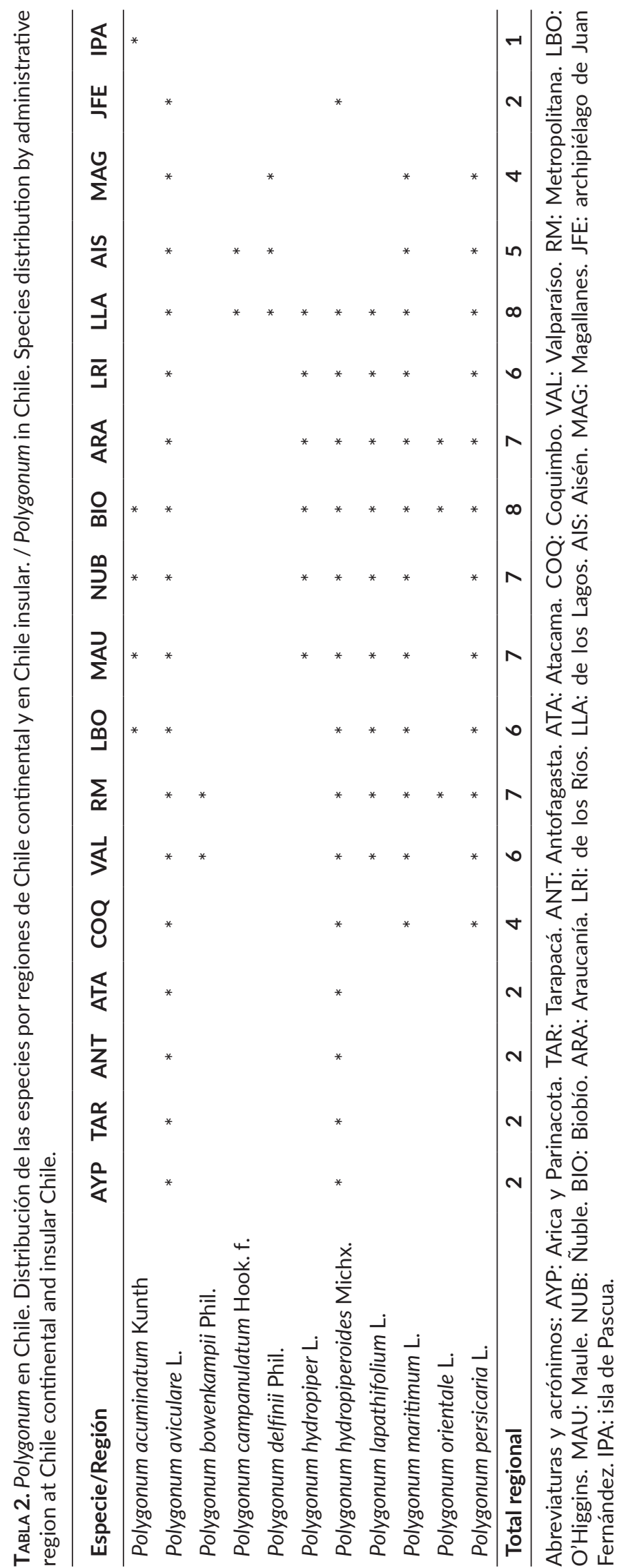

\section{RESULTADOS}

Como resultado de este trabajo se aceptan para Chile once especies de Polygonum (Tabla 1). La distribución de ellas por región se muestra en la Tabla 2. El mayor número de especies se registra entre las regiones de Valparaíso y de los Lagos. Las regiones del Biobío y de los Lagos muestran el mayor número de especies, ocho; en tanto que las regiones del extremo norte muestran las riquezas más bajas, con solo dos especies; en el territorio insular se registran especies de Polygonum en el archipiélago de Juan Fernández (P. aviculare y $P$. hydropiperoides) y en la isla de Pascua (P. acuminatum).

\section{Descripción del género en Chile}

Polygonum L. Sp. PI. 1: 359. 1753. Hierbas anuales o perennes $\mathrm{o}$, raras veces, subarbustos. Plantas terrestres o algo anfibias. Tallos erguidos o postrados, generalmente estriados, glabros, con tricomas rígidos y agudos, papilosos o con aguijones retrorsos. Hojas sésiles, subsésiles o pecioladas, alternas, glabras, con tricomas rígidos y agudos, papilosas o con aguijones retrorsos. Ócreas hialinas, bífidas cuando jóvenes y luego laciniadas, truncadas, glabras o con pelos o con cilios en su cuerpo o en el margen. Flores pediceladas, dispuestas en cimas (cincinos) en la axila de las brácteas foliosas las que, a veces, se reducen mucho y quedan representadas solo por una bractéola; en ocasiones, por la reducción de los entrenudos, las cimas se reúnen en tirsos espiciformes, laxos o densos, continuos o interrumpidos. Flores hermafroditas, actinomorfas; perigonio petaloide, algo acrescente, con (4-)5-(6) tépalos blancos, rosados o rojizos, la parte basal interna con un disco glanduloso más o menos desarrollado. Androceo (3)-6-9, estambres inclusos o exertos, insertos en distintas alturas, algunos en las comisuras de los segmentos del perigonio y otros en su parte inferior, algunas veces entre las sinuosidades del disco glanduloso; filamentos desiguales, con menos frecuencia iguales, filiformes, subulados o muy dilatados en la base; anteras elipsoides u ovoides. Gineceo con ovario sublenticular, ovoide-comprimido, ovoidetrígono u obovoide-trígono, rara vez tetrágono y muy excepcionalmente pentágono; unilocular, con 2-3 estilos soldados en mayor o menor grado o libres, por excepción cinco; estigmas capitados. Fruto una nuez lenticular o trígona, muy rara vez tetrágona y muy excepcionalmente pentágona, lisa, finamente punteada o suave hasta visiblemente estriada, parda, castaña o negra, opaca o brillante, cubierta por el perigonio o algo exerta, estilos caducos o persistentes. 
Clave para determinar las especies de Polygonum en Chile

1. Plantas leñosas o subleñosas.

4. P. campanulatum

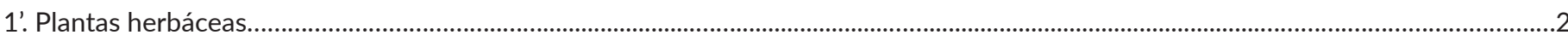

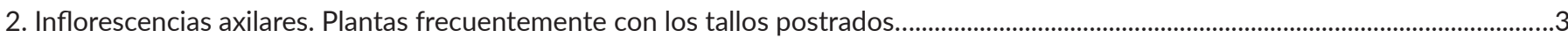

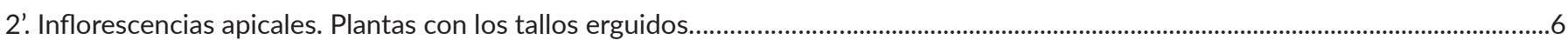

3. Hierbas anuales, sin rizomas; a veces, la raíz principal es pivotante y muy robusta. Hojas homomorfas o heteromorfas en la misma planta. Plantas ruderales o malezas de cultivos.....

2. P. aviculare

3'. Hierbas perennes, con rizomas. Hojas solo homomorfas en la misma planta. Plantas ni ruderales ni malezas de cultivos.

4. Hojas consistentemente oblongas; las nuevas fuertemente revolutas.

....5

4'. Hojas lanceoladas a lineares; las nuevas, algo revolutas.

5. Ócreas de 8-20 mm, en los tallos bien desarrollados, casi o del largo del entrenudo. Hojas verde-cenicientas. Nuez lisa. Plantas del litoral.

6. P. maritimum

5'. Ócreas de hasta $8 \mathrm{~mm}$, en los tallos desarrollados más cortas que los entrenudos. Hojas verdes, no cenicientas. Nuez estriada. Plantas de las cordilleras

3. P. bowenkampii

6. Plantas de hasta $2 \mathrm{~m}$ de altura. Tricomas no apegados al tallo. Hojas con pecíolos de 2-10 cm de longitud; lámina ovada hasta cordada, de hasta $20 \times 3-10 \mathrm{~cm}$. Plantas ornamentales ocasionalmente escapadas del cultivo.

10. P. orientale

6'. Plantas menores raras veces de más de $1,5 \mathrm{~m}$ de altura. Tricomas normalmente apegados al tallo. Hojas con pecíolos de menos de $2 \mathrm{~cm}$ de longitud; lámina lanceolada, de hasta $5 \times 1 \mathrm{~cm}$. Plantas silvestres.

7. Ocréolas (brácteas) acuminadas. Hojas con glándulas densas en el envés, a veces, solo tomentosas o apenas con tricomas adpresos. Ócreas foliares enteras o dentadas, raras veces ciliadas, glabras en la superficie.

8. P. lapathifolium

7'. Ocréolas (brácteas) no acuminadas. Hojas sin glándulas, algunas veces con tricomas y punteaduras en el envés. Ócreas foliares ciliadas en el ápice; glabras o híspido-pilosas en la superficie.

8. Tallos densamente pilosos. Las ócreas, de color marrón claro, sobrepasan generalmente la mitad del entrenudo.

1. P. acuminatum

8'. Tallos glabros o poco pilosos. Las ócreas hialinas, rara vez marrones, no alcanzan la mitad del largo del entrenudo..........9

9. Perigonio con 4 tépalos verdosos, algo rosados hacia el ápice. Perigonio y ócreas con glándulas puntiformes de color pardo-rojizo. Hojas con sabor picante.

6. P. hydropiper

9'. Perigonio con 5 tépalos rosados o raras veces blancos. Perigonio y ócreas sin glándulas puntiformes de color pardorojizo. Hojas no picantes

10. Tirsos no densos, las flores a veces se alternan en el eje. La inflorescencia raras veces de más de $4 \mathrm{~mm}$ de ancho. 7. P. hydropiperoides

10'. Tirsos densos, a veces no se distinguen sus ejes. Inflorescencia con frecuencia de más de $4 \mathrm{~mm}$ de ancho...

11. Ócreas foliares y ocréolas (brácteas) con cilios gruesos en el margen superior y sobre la superficie. Aquenios más frecuentemente lenticulares. Hierbas anuales, sin rizomas

11. P. persicaria 11'. Ócreas foliares y ocréolas (brácteas) con cilios finos, o las ócreas carentes de cilios. Aquenios trígonos, raras veces lenticulares. Hierbas perennes, con rizomas

7. P. hydropiperoides

\section{DESCRIPCIÓN DE LAS ESPECIES}

1. Polygonum acuminatum Kunth, Nova Gen. Sp. (quarto ed.) 2: 178. 1817[1818]. Persicaria acuminata (Kunth) M. Gómez, Anales Inst. Seg. Enseñan. 2: 278. 1896.

TIPO: Venezuela, "Bordones in humidis", 9-10-1799, Humboldt \& Bonpland 135 (holotipo, BW cb 07642010 Fotografía!).

Hierba perenne. Tallos erguidos, de $60-200 \mathrm{~cm}$ de alto y 3 $\mathrm{mm}$ de diámetro, estriados, densamente pilosos hacia el extremo superior. Hojas alternas, brevemente pecioladas, glabras a seríceas, anchamente lanceoladas, de (6)7-15 (20) $x(0,8)$ 2,5-4 cm; envés y margen cubiertos de pelos rígidos; margen apenas ondulado, base atenuada y ápice acuminado. Ócreas foliares turbinadas, de $15 \mathrm{~mm}$ de largo, truncas, con cilios en los bordes y en la lámina; en la parte inferior del tallo la cubierta de pelos de hasta $1,5 \mathrm{~mm}$ de largo dificulta su observación. Brácteas de 2-3 mm de largo. Inflorescencias en tirsos espiciformes densos, no interrumpidos, de 5-6,5 cm de largo, formados por cimas (cincinos) de 5 flores. Perigonio con 4(5) tépalos blancos con tinte rosado; androceo 6, anteras amarillas de 0,5 mm de largo; gineceo con ovario de 3-4 mm 
de largo, con 2 carpelos y 2 estilos de $2-3 \mathrm{~mm}$ de largo. Fruto nuez lenticular, de 2-3 mm de largo, negra, brillante, globosa (Fig. 1).

Nombre común: Tavari (isla de Pascua)

Distribución geográfica: especie alóctona asilvestrada. En Chile continental se la ha coleccionado en las regiones de O'Higgins, Maule, Ñuble y Biobío; en Chile insular, en la isla de Pascua (Rodríguez et al., 2018). La especie es originaria de un territorio que comprende México, parte de Sudamérica y las Antillas (Stevens et al. 2001).

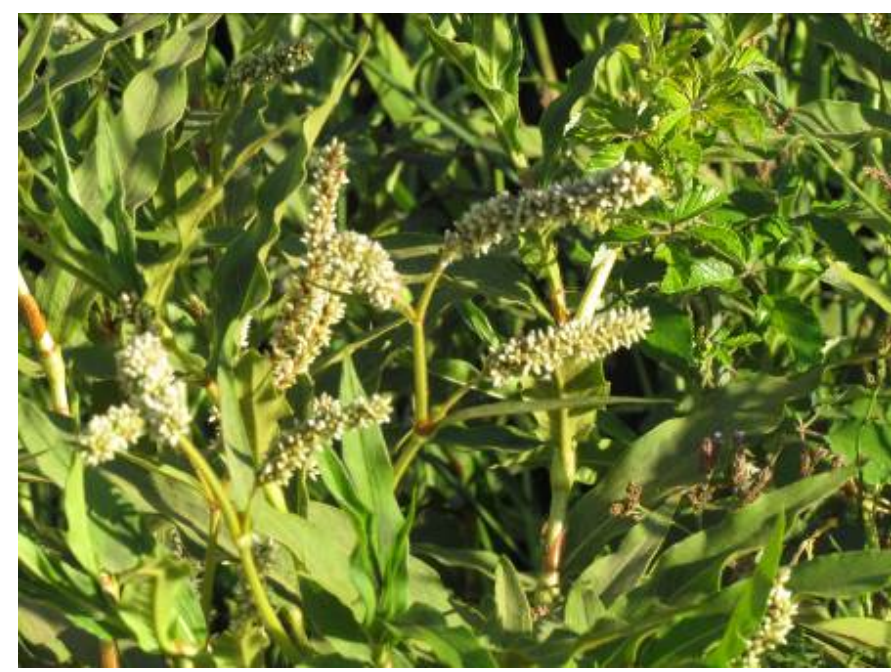

Figura 1. Polygonum acuminatum Kunth. Inflorescencias. / Inflorescences. Fotografía: S. Teillier.

Observación: Matthei (1995) afirma que en Chile continental es una especie que crece en los canales de regadío y en los arrozales.

Materiales estudiados: CHILE. Región de Valparaíso: prov. Marga Marga, Limache, cuesta La Dormida, 3-IV-1972, M. Bassano s/n (SGO 138693). Isla de Pascua, 24-V-1956, J. Israel $\mathrm{s} / \mathrm{n}$ (CONC 19789). Rano Aroi, in and around the crater, 26-I-1988, T.F. \& P.G. Stuessy 11023 (CONC). Región de O'Higgins: prov. Colchagua, Santa Cruz, VI-1983, S. Contreras s/n (SQF 16015). Región del Maule: prov. Curicó, Chomedahue I-1878, Sanfurgo s/n (SGO 48024). Palquibudi, camino de Rauco a Hualañe, 70 m s.n.m., 29-XI-1990, O. Matthei \& M. Quezada 1225 (CONC, SQF). Prov. Talca, Colín, III-1963, A.M. Bravo (CONC 133964). Predio Venecia, 1 km al sur de Talca, en cultivo de arroz, 16-III-1988, 100 m s.n.m., R. Rodríguez \& M. Baeza 2341 (CONC). Región Ñuble: prov. Itata, Portezuelo, 7-VI-1954, M. Ledezma 447 (SQF). Prov.
Diguillín, Chillán, estación Quilamapu, INIA, 135 m s.n.m., 21-I-1991, O. Matthei \& M. Quezada 1410 (CONC). Región del Biobío, prov. Concepción, Villamávida, 25-II-1945, E. Kausel 1602 (SGO). Concepción, km 25, camino a Florida, 2-IV-1982, L. Landrum 4430 (SGO). Prov. Biobío, camino a Santa Bárbara, $1 \mathrm{~km}$ desde el longitudinal, $150 \mathrm{~m}$ s.n.m., 12-III-1976, C. Marticorena, O. Matthei \& R. Rodríguez 871 (CONC). Región de la Araucanía: prov. Cautín, Cunco, 3-II1937, H. Gunckel s/n (SQF (11013). Región de los Ríos: prov. de Valdivia, Valdivia, XI-1926, H. Gunckel s/n (SQF 12198).

2. Polygonum aviculare L., Sp. PI. 1: 362. 1753. TIPO: "Habitat in Europae cultis ruderatis" "15 aviculare" (LINN).

Polygonum striatum K. Koch, Linnaea 22: 206. 1849. TIPO: "Chile", B. V. Besser s/n. (holotipo, B cb 100248293 Fotografía!). Polygonum arenastrum Boreau, Flore du Centre de la France (ed. 3) 2: 559. 1857. TIPO: "France: Cabourg (Calvados, Normandie), 28 août 1850" A. Boreau s.n. (ANG-Fotografía!).

Polygonum caballeroi Phil., Anales Univ. Chile 81: 491. 1892. TIPO: Chile, "prope Ovalle invenit Januario 1880 ornat.", Amable Caballero s/n. (lectotipo aquí designado, SGO 48027!; isolectotipo, SGO 38704!).

Nombre común: Pasto del pollo, sanguinaria

Hierba anual, a veces con raíces muy gruesas y que se tornan algo leñosas. Tallo muy ramoso, procumbente hasta horizontal, de hasta $60 \mathrm{~cm}$ de alto o de longitud. Hojas (filotaxis), subsésiles, anchamente elípticas a lanceoladas, de 5-40 x 2(4)-8 (18) mm, homomorfas o heteromorfas; margen entero y ápice agudo. Ócreas foliares hialinas, acuminadas o, con el tiempo, laciniadas, de hasta $10 \mathrm{~mm}$ de largo. Inflorescencias en cimas axilares (cincinos) con 2-5 flores. Ocréolas de 1-5 $\mathrm{mm}$ de largo. Perigonio 5, de 2,5-3 mm de largo, con 3 tépalos externos y 2 internos, de color blanco o rosado, tubo basal de hasta un tercio de la parte libre de los tépalos; androceo 8; ovario con 3 estilos, de 0,14-0,25 mm de largo, incluyendo el estigma. Fruto nuez ovoide, de 1,1-3 mm de largo, trígona, pardo-anaranjada a negruzca, punteada o finamente estriada (Fig. 2).

Distribución geográfica: especie alóctona asilvestrada. En Chile se la encuentra desde la Región de Arica y Parinacota hasta la de Magallanes y en la isla Masatierra (Robinson Crusoe) del archipiélago de Juan Fernández. Tiene una amplia distribución en sitios alterados, ruderales y urbanos y en la agricultura se la considerada como una maleza (Matthei, 1995). Especie de origen eurasiático, de amplia distribución en el mundo. 


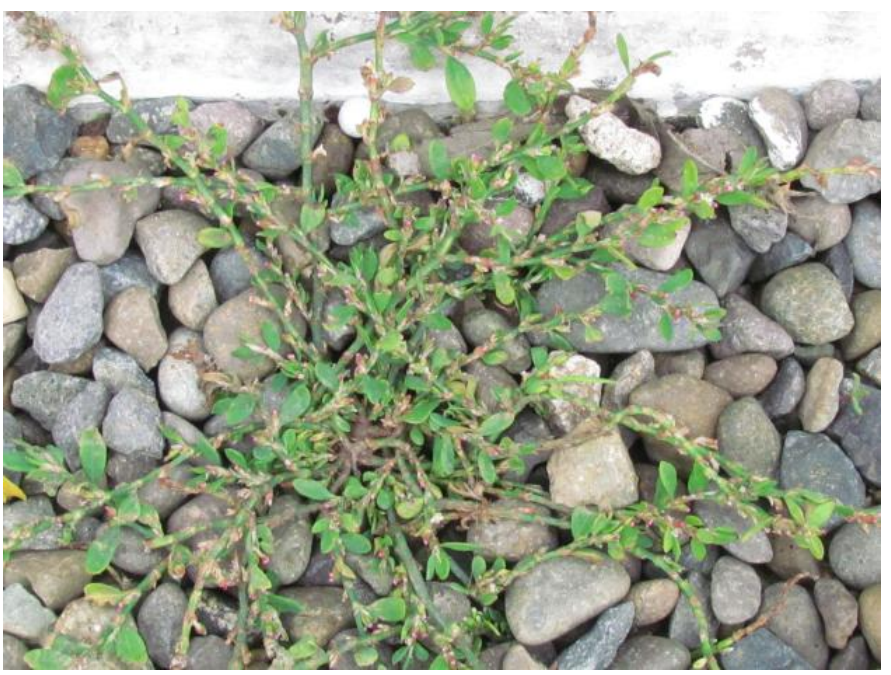

Figura 2. Polygonum aviculare L. Hábito. / Habit. Fotografía: S. Teillier.

Observaciones: especie anual, bienal o perenne de vida corta, extremadamente variable en su morfología (Cialdella, 1989). En Chile se encuentran desde formas con raíces muy gruesas, casi rizomatosas, hasta pequeñas plantas anuales; existen formas erectas o tendidas, heterófilas y homófilas. Las condiciones en que crece influyen mucho en el tamaño y en el hábito; en sitios agrícolas bien regados y con disponibilidad de nutrientes se encuentran ejemplares de gran tamaño; además, existe una variación estacional en la forma de las hojas y su densidad en las ramas. Polygonum arenastrum es una forma homófila frecuentemente considerada como una especie diferente (Webb \& Chatter 1964, Mertens \& Raven 1965, Marticorena \& Quezada, 1985, Villar 1990), pero, siguiendo el criterio de Cialdella (1989), de Costea \& Tardif (2004) y de Rodríguez et al. (2018) se la ha incluido en la sinonimia de P. aviculare. Polygonum caballeroi representa una forma robusta y algo leñosa de P. aviculare, por ello se la incluye entre sus sinónimos, pero el material conocido es escaso. Además del ejemplar tipo se conocen unos pocos ejemplares, e.g. uno coleccionado en Corral de Julio, provincia del Choapa, Región de Coquimbo, por M. Muñoz 885 (SGO), y otro en el humedal de Batuco por Sparre (10959, CONC). Se lo ha lectotipificado eligiendo el material más completo y con las etiquetas más legibles. Polygonum striatum, de acuerdo con Cialdella (1989) correspondería a un sinónimo de $P$. aviculare, criterio que se sigue en la presente contribución.

Materiales estudiados: CHILE. Región de Arica y Parinacota, prov. Putre, valle de Codpa, 2000 m s.n.m., 15-III-1948, F. Sudzuki 621-A (SGO). Provincia Arica, quebrada Vítor, Chaca,
22-IX-1955, M. Ricardi 3450 (CONC). Región de Antofagasta, Prov. Antofagasta, $12 \mathrm{~km}$ al norte de Antofagasta, salar El Carmen, 390 m s.n.m., M. Baeza, Aqueveque \& Kottirsch 667 (CONC). Prov. El Loa, camino Calama-Toconce, 6-XII1957, L. E. Navas 2416 (SQF), San Pedro de Atacama, 2440 m s.n.m., 19-I-1993, G. Baumann s/n (CONC 129501). Región de Atacama, prov. Copiapó, fundo Toledo, $15 \mathrm{~km}$ al norte de Copiapó, 285 m s.n.m., 25-III-1987, O. Matthei \& R. Rodríguez, 456 (CONC). Prov. Huasco, valle de San Félix, 1100 m s.n.m., 16-XII-1941, E. Pisano \& R. Bravo 1109 (CONC). Región de Coquimbo, prov. Elqui, cerro oeste canchas de esquí, 1200-1500 m s.n.m., 6-I-1988, F. Squeo, 88014 (CONC). Prov. Limarí, Ovalle, quebrada Ingenio, 200 m s.n.m., 2-X1949, C. Jiles 1477 (CONC). Prov. Choapa, Corral de Julio, Aguas Malas. 6-II-1976, M. Muñoz 855 (SGO). Región de Valparaíso, prov. Valparaíso, Viña del Mar, paseo costero, 0908-2014, S. Teillier \& P. Novoa 8002 (CONC). Quintero, El Durazno, 2-XI-1952. H. Gunckel 24359 (SQF). Viña del Mar, 2-I-1984, M. Arriagada s/n (SQF 6872). Juan Fernández, isla Robinson Crusoe (Masatierra), cerro El Yunque, I-II-1967, H. Landman (s/n) (SGO 130694). Between the cemetery by San Juan Bautista and the beach, $5 \mathrm{~m}$ s.n.m., 25-XI-1980, T. Stuessy, O. Matthei, R. Sanders, H. Valdebenito 5446 (CONC). Masafuera (Alejandro Selkirk), in and around small village at mouth of quebrada Casas, 5 m s.n.m., 23-I-1984, T. Stuessy, D. Crawford, P. Pacheco, H. Valdebenito, E. Ruiz \& A. Landeros, 6355-a. Prov. Marga Marga, Marga Marga, I-1911, Jaffuel (CONC 46500). Prov. Los Andes, cerro de La Virgen, Los Andes. XI-1954, M. Arriagada 198 (SQF). Prov. San Antonio, fundo Colegio de Bucalemu, 60 m s.n.m., O. Matthei \& M. Quezada 282 (CONC). Región Metropolitana, prov. Chacabuco, Peldehue, 14-V-1988, X. Escalona s/n (SQF 17100). Batuco, 4-IV-1954, M. Arriagada (SQF 6412). Prov. Cordillera, El Alfalfal, I-1888, R. Philippi s/n (SGO 48077). San Alfonso, 11-I-1954, M. Arriagada s/n (SQF 9076). San José de Maipo, 8-IV-1986, V. del Villar s/n (SQF 16515). Pirque, 9-X-1982, C. Tapia s/n (SQF 16012). Prov. Santiago, Quilicura prope Santiago, III-1861, F. Philippi s/n (SGO 48068). Cerro San Cristóbal, 21-XII-1953, L.E. Navas 503 (SQF). Cerro San Cristóbal, 14-05- 1954, L.E. Navas 677 (SQF). Cerro San Cristóbal, 18-04- 1954, L.E. Navas 587 (SQF). Macul, 25XI-1961, M.A. González s/n (SQF 14377). Maipú, II-1955, L. Fuenzalida s/n (SQF 9196). Ñuñoa, 6-I-1954, M. Moreno s/n (SQF 14376). Providencia, 13-I-1954, M. Arriagada s/n (SQF 9641). San Joaquín, 30-I-1988, P. Rivas s/n (SQF 17099). Santuario de la naturaleza Yerba Loca, borde del estero Yerba Loca, cerca de la confluencia con el río San Francisco, 1800 m s.n.m., 16-X-1999, M. Kalin-Arroyo, C. Valdivia \& P. Mc. Pherson, 994780 (CONC). Farellones, en el pueblo, $2465 \mathrm{~m}$ s.n.m., 7-III-2007, M. Mihoc 744 (CONC). Prov. Talagante, 
Padre Hurtado, 12-04-1986, D. Lenac s/n (SQF 16502). Prov. Maipo, cerro Lo Chena, San Bernardo, 550 m s.n.m., 10-X1950, L. Recabarren 226 (SQF 4447). San Bernardo, 17-041987, L. Ravanales s/n (SQF 16839). Región de O'Higgins, prov. Cachapoal, fundo Totihue, Requinoa, II-1952, J. Petit $\mathrm{s} / \mathrm{n}$ (SQF 4403). Prov. Colchagua, vegas del Flaco, 12-II-1955, M. Ricardi 3241. San Fernando, Las Rosas de Antivero, $385 \mathrm{~m}$ s.n.m., 28-XI-1989, O. Matthei \& M. Quezada 657 (CONC). Prov. Cardenal Caro, Tanumé, loma El Maitén, 350 m s.n.m., 25-I-2005, I. Vera \& N. Ortiz 44 (CONC). Región de Maule, prov. Curicó, Curicó, I-1952, A. Ampuero s/n (SQF 4438). Molina 21-05-1981, I. Escobar s/n (SQF 15392). Prov. Talca, predio Venecia, $1 \mathrm{~km}$ al sur de Talca, en cultivo de arroz, $100 \mathrm{~m}$ s.n.m., 16-III-1988, O. Matthei \& M. Baeza 2338. Prov. Linares, Villa Alegre, III-1939. M. Espinosa s/n (SGO 65666). Región de Ñuble, prov. Diguillín, Chillán 30-III-1986, R. Martínez s/n (SQF 16503). Fundo San José, camino a San Nicolás, 29-I1959, O. Matthei s/n (CONC 27126). Prov. Itata, Cobquecura, 7-IV-1954, M. Ledezma 259 (SQF 11829). Región del Biobío, in arenis Nacimiento, provincia Conceptionis, IV-1838, C. Gay, 2038 (SGO). Prov. Biobío, fundo Santa Olga, 265 m s.n.m., II-1954, E. Gautier s/n (CONC). Los Ángeles, 8-IV-1972, E. Oehrens 1090 (CONC). Prov. Concepción, Concepción, calle Paicaví entre San Martín y Cochrane, 10 m s.n.m., 4-12-1988, P. López, 290 (CONC). Tomé, quebrada California, 24-XII1944, C. Junge s/n (CONC 5244). Región de la Araucanía, prov. Cautín, Toltén, 10-15 m s.n.m., 1-III-1942, H. Gunckel 12839 (SQF). Región de los Ríos, prov. Valdivia, Llancacura, 21-XII1947, H. Gunckel 17305 (SQF). Neltume, reserva biológica Huilo Huilo, pampa Bandurrias, 545 m s.n.m., 14-01-2012, S. Teillier, J. Delaunoy, J. Macaya \& C. Bonnemaison, 7339 (CONC). Región de los Lagos, prov. Osorno, islote Rupanco, 7-III-1979, R. Godoy (SGO 104574). Pichipicha, entrada a Quilacahuín, 45 m s.n.m., 19-III-1990, O. Matthei \& M. Quezada, 1028 (CONC). Prov. Llanquihue, lago Llanquihue, Punta Larga, 50 m s.n.m. 5-II-1953. A. Bórquez s/n (SQF 8767). Puerto Montt, II-1952, E. Labarca s/n (SQF 4436). Prov. Chiloé, lago Huillinco, Chonchi, 9-10 III-1942, E. Pisano \& S. Zavieso 1373 (SGO). Isla Talcán, sector sur, 17 m s.n.m., 25-I-1985, C. Villagrán \& C. Águila, 6241 (CONC). Región de Aysén, prov. Aysén, Coyhaique, 7-II-1934, M. Espinosa s/n (SGO 70316). Puerto Aysén, 18-II-1967, T. Seki 165 (CONC). Prov. Capitán Prat, Cochrane, lago Esmeralda, 310 m s.n.m., I-2007, C. Ramírez \& O. Vidal s/n (CONC 166635). Región de Magallanes, Prov. Última Esperanza, sierra Los Baguales. Estancia La Cumbre. Lecho del río Baguales, junto a las casas. 550 m s.n.m., 30-I-1987, A. Landero 810 (CONC). Puerto Natales, calles de la ciudad, 5 m s.n.m., 24-I-1988, O. Matthei \& R. Rodríguez 642 (CONC). Prov. Magallanes, Santa María, Agua Fresca, 26 km S de Punta Arenas, 18-II-1945, W.
Biese 1145 (SGO). Punta Arenas, fuerte Bulnes km 39, 5 m s.n.m., 21-I-1988, O .Matthei \& R. Rodríguez 557 (CONC). Prov. Tierra del Fuego, Tierra del Fuego, I-1966, T. Tsujii 450 (CONC). Porvenir, estancia Las Mercedes, 15 m s.n.m., 8-VI2004, N. Véjar (SAG) 1853 (CONC).

3. Polygonum bowenkampii Phil., Linnaea 33: 224. 1864. TIPO: Chile. "In Andibus prov. Santiago primus legit ornat. Guillem. Bowenkamp, horto amici Segethi praefectus, deinde et ipse frequens inveni", (holotipo, SGO 48075! isotipo, B cb 100248258 Fotografía!). Hierba perenne, provista de un rizoma vertical. Tallos horizontales, glabros, de hasta unos $15 \mathrm{~cm}$; entrenudos más largos en las ramas vegetativas que en las florales. Hojas glabras, aunque el envés presenta pequeñas punteaduras, verdes, no cenicientas, con 2-3 pares de nervaduras, estrechamente lanceoladas, de 5-10 × 2-4 mm, más cortas en el ámbito de las inflorescencias, agudas; ócreas hialinas, de hasta $8 \mathrm{~mm}$ de longitud, más cortas o apenas del largo de los entrenudos, enteras, en las ramas vegetativas, en las floríferas, pierden el ápice, la base adquiere un color marrón y son fimbriadas o ciliadas. Flores dispuestas en cimas cortas, axilares, casi sésiles. Perigonio de $2-3 \mathrm{~mm}$ de longitud, 3 tépalos externos rosados, con la base del envés verde y 2 , internos, blancos, tubo de hasta un tercio de la parte libre de los tépalos; androceo con 8 estambres; ovario con 3 estilos estos, incluyendo el estigma, de 0,25-0,33 mm de largo. Nuez ovoide, pardo-anaranjada a negruzca, punteada o finamente estriada, de (2) 3-4 mm de longitud, trígono o tetrágono, caras cóncavas (Fig. 3A y 3B).

Distribución geográfica: especie endémica de las cordilleras de los Andes y de la Costa de las regiones de Valparaíso y Metropolitana, donde crece hasta unos $2800 \mathrm{~m}$ de altitud. Más frecuente en los sitios perturbados.

Observación: esta especie, por su carácter de hierba perenne, se la ha relacionado con Polygonum maritimum L., con la que comparte algunos caracteres anatómicos como los tricomas escutiformes y los cilios (R. Peña \& I. Latorre, datos no publicados). En la clave del género se mencionan los principales caracteres morfológicos que las diferencian; $P$. maritimum es una especie del litoral, bien ramificada, con los tallos largos y P. bowenkampii, una planta de las cordilleras poco ramificada y con tallos más cortos y apegados contra al suelo.

Materiales estudiados: CHILE. Región de Valparaíso, prov. Los Andes, río Colorado, vegas de Lagunillas, 3200 m s.n.m., 14-01-2010, S. Teillier \& J. Delaunoy 7661 (CONC). Región 
Metropolitana, prov. Chacabuco, Altos de Tiltil, IV-1895, R.A. Philippi s/n (SGO 48064). Prov. Cordillera, laguna Negra, II-1901, Oyarzún s/n (SGO 4065). Valle de Morales, en la parte baja del valle hasta la laguna de Morales, Lo Valdés, 20-XI-1940, A. Garaventa 5704 (CONC). Parque nacional El Morado, 2090 m s.n.m., 29-XII-1990, S. Teillier \& L. González 2438 (SGO). Reserva nacional Río Clarillo, II-2004, F. Romero \& G. Aldunate 64 (CONC). Centro de esquí Lagunillas, $2300 \mathrm{~m}$ s.n.m., 27-01-2009, S. Teillier, F. Romero, I. Goic \& X. Romero 5616-B (CONC). Prov. Santiago, Lo Barnechea, cordillera de Las Arañas, I-1861, Landbeck s/n (SGO 48076). Subida a Potrero Grande, 2160 m s.n.m., 8-12-1933, K. Behn (CONC 3314). Farellones, camino a Valle Nevado, 2850 m s.n.m., 9-I1993, M. Muñoz \& U. Egli 3208 (SGO). (Centro de esquí) La Parva, 2765 m s.n.m., 5-I-1979, M. Muñoz \& I. Meza 1296 (SGO). Los Azules, 2250 m s.n.m., 20-XI-1955, F. Schlegel 903 (CONC). Santuario de la naturaleza Yerba Loca, vegas en el estero Yerba Loca, sector La Polvareda, 2630 m s.n.m., M.T. Kalin Arroyo \& A.M. Humaña 991823 (CONC).
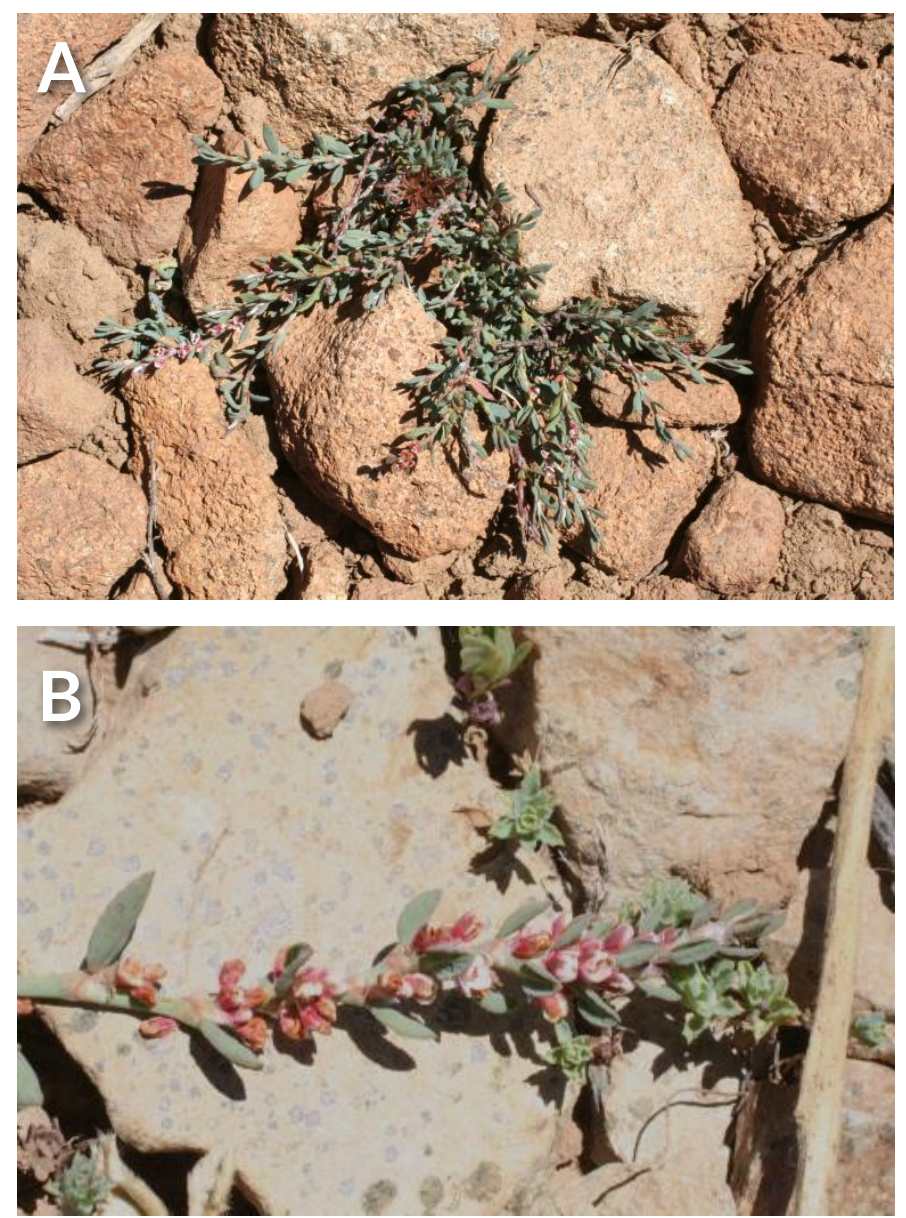

Figura 3. Polygonum bowenkampii Phil. A) hábito, B) hojas y flores. ( A) habit, B) leaves and flowers. Fotografías: S. Teillier.
4. Polygonum campanulatum Hook. f., Fl. Brit. India, 5 (13): 51. 1886. Persicaria campanulata (Hook. f.) Ronse Decr., Bot. J. Linn. Soc., 98(4): 367. 1988. Koenigia campanulata (Hook. f.) T.M. Schust. \& Reveal, Taxon 64(6): 1200-1201. 2015. TIPO: India, "above Jhuni, Kumaon, 7500 ft", A. Strachey \& Winterbottom (lectotipo, K cb 000831211 Fotografía! designado en Hong, 1992; isolectotipo, GH cb 00036778 Fotografía!). Subarbusto subleñoso, de $60-150 \mathrm{~cm}$ de alto, glabro o pubescente, profusamente ramificado. Hojas densamente cubiertas de pelos, con pecíolo 3-5 mm de largo, elípticas a lanceoladas, de 5-10 x 1,5-3,5 cm, acuminadas, base angosta, cara inferior verde claro con la nervadura sobresaliente, la superior, verde oscura, pubescente con la nervadura no sobresaliente; ócreas foliares de 8-10 mm de largo, anchas, caducas. Flores numerosas, dispuestas en tirsos espiciformes, de $2-5 \mathrm{~cm}$ de largo; tépalos rosados. Androceo con 8 estambres. Ovario con 2-3 estilos. Nuez trígona, de $2 \mathrm{~mm}$ de largo (Fig. 4).

Distribución geográfica: especie alóctona asilvestrada. Matthei (1985) la refirió como una maleza en la provincia de Chiloé; Rodríguez et al. (2018), a su vez, la señalan como una especie naturalizada en las regiones de los Lagos y de Aysén.

Observación: especie ornamental, de origen asiático, escapa del cultivo.

Materiales estudiados: CHILE. Región de los Lagos, Prov. Osorno, La Gruta, camino de Osorno a Puerto Octay, $185 \mathrm{~m}$ s.n.m., 20-III-1990, O. Matthei \& M. Quezada 1103 (CONC).

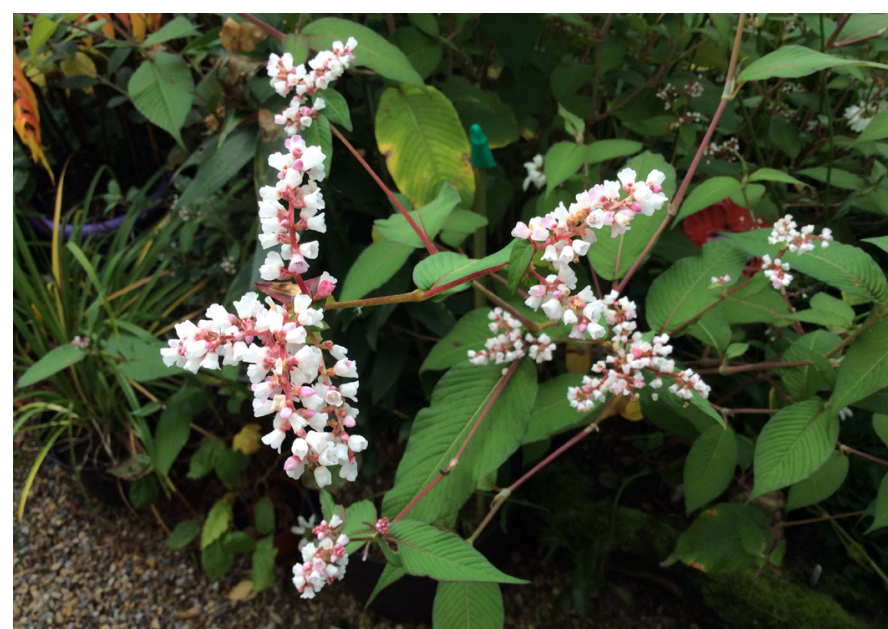

Figura 4. Polygonum campanulatum Hook. f. Inflorescencias. / Inflorescences. Fotografía: J. Macaya-Berti. 
Prov. Llanquihue, alto Puerto Varas, 150 m s.n.m., 18-III1990, O. Matthei \& M. Quezada 992 (CONC). Prov. Chiloé, El Quilar, camino de Chacao a Ancud, 30 m s.n.m., 14-III-1990, O. Matthei \& M. Quezada 823 (CONC). Coinco Bajo $25 \mathrm{~m}$ s.n.m., 16-III-1990, O. Matthei \& M. Quezada 904 (CONC).

5. Polygonum delfinii Phil. Anales Univ. Chile 91: 490. 1895. TIPO: Chile. "In valle fluminis Palena 1887 ab orn. Friderico Delfin repertum" (holotipo, SGO 38699!).

Hierba perenne, de 25-30 de altura. Tallos postrados, estriados; entrenudos superiores del largo de la ócreas foliares. Hojas glabras, con pecíolos de 1,2 mm de largo, lámina oblonga, conduplicada (revoluta), las mayores, extendidas, de $6 \mathrm{~mm}$ de largo; ócreas foliares laciniadas con las nervaduras bien marcadas. Inflorescencias en cimas (cincinos) axilares, con 5-7 flores; perigonio de 0,9 $\mathrm{mm}$ de largo. Androceo con los estambres con anteras amarillas, de 0,3 $\mathrm{mm}$ de largo. Ovario con 3 estilos. Nuez ovoide, trígona en el ápice, acuminada, pardo-anaranjada, lisa y lustrosa, de 2,3 mm de largo (Fig. 5).

Distribución geográfica: especie endémica del litoral sur de Chile, con distribución geográfica conocida entre las regiones de los Lagos y de Magallanes. Crece en sitios aledaños al litoral inclusive en algunos que de acuerdo con las etiquetas de los materiales son inundados por las mareas altas.

Observación: si bien Marticorena \& Quezada (1985) la consideran como una buena especie, no figura en el catálogo de Rodríguez et al. (2018). Es muy afín a Polygonum maritimum L. y quizás sea parte de su variabilidad, pero su eventual sinonimización requiere de más colecciones y un estudio más detallado.

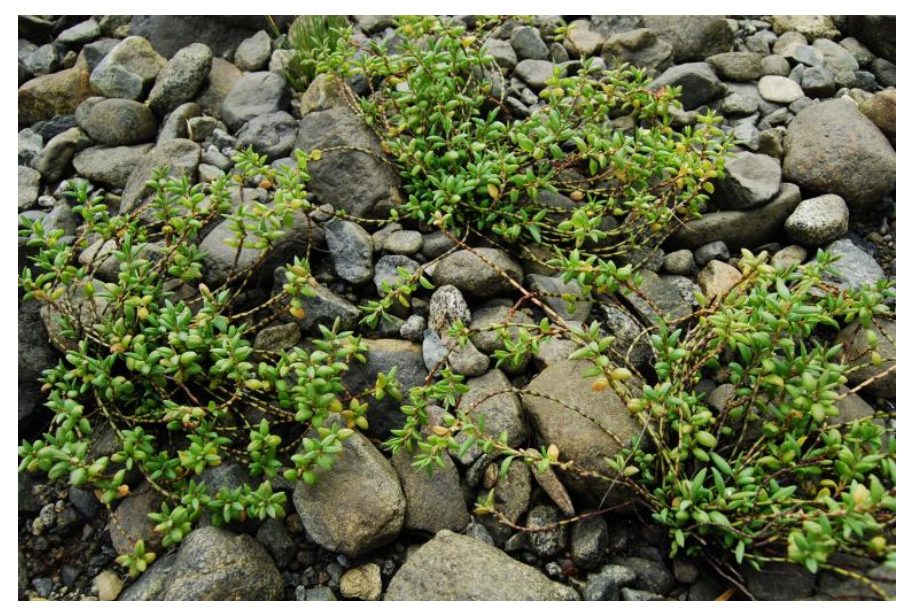

Fıgura 5. Polygonum delfinii Phil. Hábito. / Habit. Fotografía: M.V. Legassa.
Materiales estudiados: CHILE. Región de los Lagos, prov. Llanquihue, Cochamó, II-1930, D. Rudolf 17 (SGO). Prov. Chiloé, Palena, Hornopirén, San Ignacio de Huinay, J. L. Fernández, L. Medina, R. Morales, F. Muñoz, RM 2725 (CONC, SGO). Región de Aysén, prov. Aysén, Puerto Puyuhuapi, 23XII-1939, H. Schwabe 43 (CONC). Laguna San Rafael, rio Témpanos, desaguadero de la laguna, 16-I-1959, C. Muñoz 6194 (SGO). Laguna San Rafael, playa en el istmo de Ofqui, 23-II-1999, S. Teillier \& C. Márquez, 4344 (CONC). Región de Magallanes, prov. Magallanes, Patagonia, seno de Otway, Río Grande, 16-IV-1908, C. Skottsberg 260 (SGO).

6. Polygonum hydropiper L. Sp. PI. 361. 1753. Persicaria hydropiper (L.) Delarbre, Fl. Auvergne ed. 2: 518. 1800. TIPO: "Herb. Linn. no. 510.9 (LINN Fotografía!) designado por Reveal et al., Taxon 59: 982. 2010.

\section{Nombre común: Duraznillo}

Hierba anual, de $20-80 \mathrm{~cm}$ de altura. Tallos estriados, delgados, raras veces ramificados. Hojas casi sésiles, lanceoladas a ovado-lanceoladas, de 2-10 x 0,5-2,5 cm, enteras, ciliadas en el borde; ócreas turbinadas, truncadas, de 10-15 mm de largo, cilios marginales de 1,5-2 mm de largo; brácteas de $2 \mathrm{~mm}$ de largo reducidas a la ócrea, turbinadas, con los márgenes algo ciliados. Inflorescencias en tirsos espiciformes con el ápice nutante, de 1,5-4 cm de largo. Perigonio verdoso y algo rosado-rojizo en el ápice, de 2,5 mm de largo, tépalos 4, punteados con glándulas pardo-amarillentas muy conspicuas; androceo con 6 estambres, de 0,7 mm de largo, anteras amarillas; gineceo con ovario de $2 \mathrm{~mm}$ de largo, 2 o 3 estilos y el estigma capitado. Nuez trígona o lenticular, de 2,5-3 mm de largo, rugosa o punteada, de color pardo a negro (Fig. 6).

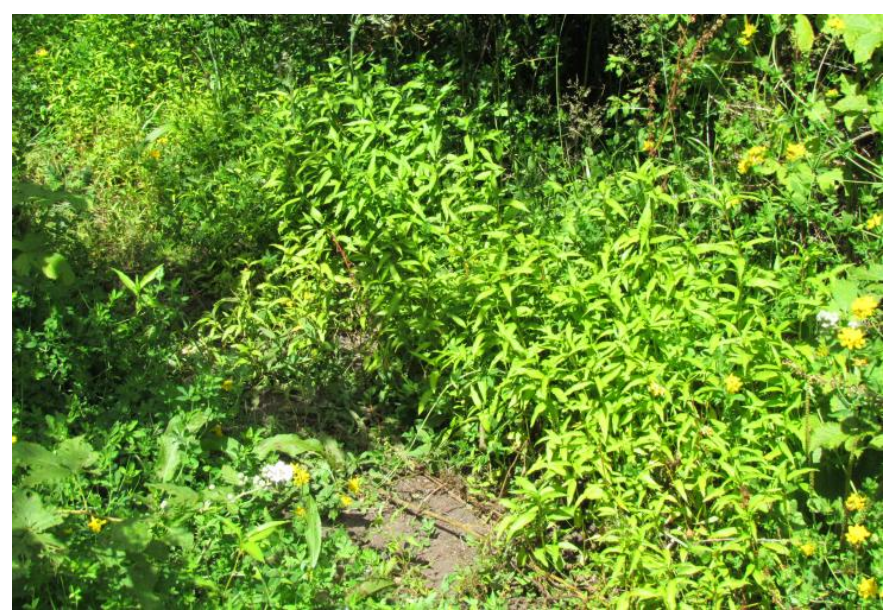

FIgURA 6. Polygonum hydropiper L. Hábito / Habit. Fotografía: S. Teillier. 
Distribución geográfica: especie originaria de Eurasia. En Chile crece como alóctona asilvestrada. Se la ha coleccionado entre las regiones de Maule y de los Lagos.

Observación: en ausencia de las partes subterráneas, las plantas de Polygonum hydropiper se asemejan a las de $P$. hydropiperoides Michx., pero se distinguen de ellas por sus flores con los tépalos marcadamente glandulosos.

Materiales estudiados: CHILE. Región de Maule, prov. Linares, a lo largo del río Achibueno, desde junta estero Las Ánimas a junta estero Riecillo, 9-III-1999, E. Ruiz \& P. López 1287 (CONC). Camino de Las Ánimas a Monte Oscuro, 9-III1999, E. Ruiz \& P. López 1342 (CONC). Región de Ñuble. Prov. Diguillín, Chillán, 3-V-1954, M. Ledezma 453 (SQF). Prov. Punilla, carretera Panamericana, $1 \mathrm{~km}$ al sur de Buli, 175 m s.n.m., 14-III-1988, R. Rodríguez \& M. Baeza 2270 (CONC). Los Ríos, Prov. Valdivia, Neltume, reserva biológica Huilo Huilo, pampa Bandurrias, 22-01-2011, S. Teillier, J. Delaunoy \& C. Bonnemaison 7401 (CONC). Región de los Lagos, prov. Osorno, barra del rio Bueno, 18-21-II-1948, B. Sparre 4549 (SGO). Islote Rupanco, III-1978, R. Godoy (SGO 104575). Pichipicha, entrada a Quilacahuín, 45 m s.n.m., 19-III-1990, O. Matthei \& M. Quezada 1025 (CONC). Chifca, camino desde la ruta 5 a Trafún, 80 m s.n.m., 20-III-1990, O. Matthei \& M. Quezada 1124 (CONC).

7. Polygonum hydropiperoides Michx., Fl. Bor. Amer. 1: 239. 1803. Persicaria hydropiperoides (Michx.) Small, Fl. S.E. U.S.: 378. 1903. TIPO. Estados Unidos de América, "Hab. In Pensylvania, Virginia, Carolina". Am. Septentr., 1795, Michaux s.n. (lectotipo, P 00734462 Fotografía! aquí designado; isolectotipos, B 10_0279365 Fotografía!, P 00734463 Fotografía!).

Polygonum persicarioides Kunth, Nov. Gen. Sp. (quarto ed.) 2: 179. 1817[1818]. Persicaria persicarioides (Kunth) Small, Fl. S. E. U.S.: 378. 1903. Polygonum hydropiperoides Michx. var. persicarioides (Kunth) Stanford, Rhodora 28: 27. 1926.

TIPO: México. "Crescit in aquis stagnantibus Regni Mexicani prope Queretaro, alt. 995 hexap.; floret junio", A.J.A. Bonpland 4599 (holotipo: P cb 00136034 Fotografía!).

Polygonum virgatum Cham. \& Schltdl., Linnaea, 3: 45. 1828. TIPO: "Brasilia meridionalis", F. Sellow d. 1559 (lectotipo B cb 100248289 Fotografía! aquí designado, isolectotipo HAL cb 0053998 Fotografía!).

Polygonum rivulare Phil., Linnaea 30: 199. 1850-1860, nom. illeg. hom., non Roxb. (1814). TIPO: Chile, "in fossis prope vallem flum. Mapocho prope Santiago legi potest", R.A. Philippi s/n (holotipo: SGO 48021!).

Nombre común: Duraznillo

Hierba perenne, rizomatosa, de hasta $60 \mathrm{~cm}$ de altura, tallo erecto, estriado, glabro en la base. Rizoma, $1,5 \mathrm{~mm}$ de diámetro. Hojas subsésiles, lanceoladas, con tricomas rígidos en el envés, de 2-11 × 0,4-2 cm, la haz glabra o pilosa; ócreas turbinadas, truncadas, membranosas, de $10-13 \mathrm{~cm}$ de largo, con fascículos de pelos adpresos, algunos ejemplares con puntos blancos en el envés de las hojas; estípulas reducidas a las ócreas, ciliadas o largamente pestañosas, con la superficie glabra o algo hirsuta. Inflorescencias en tirsos (fascículos) interrumpidos o más o menos continuos y densos (var. persicarioides), de 4-10 $\mathrm{mm}$ de ancho formados por cimas (cincinos) con 6-8 flores; ocréolas con o sin cilios; perigonio con 5 tépalos rosados, pedicelos de $3-4 \mathrm{~mm}$ de largo; androceo con 8 estambres, anteras color ocre o rosadas; gineceo con ovario de 1,5-1,8 mm de largo, con 2 estilos. Nuez trígona o, raras veces, lenticular, de color pardo claro a oscuro, brillante, de 2-3 mm de largo (Fig. 7A y 7B).

Distribución geográfica: especie americana. En Chile se la ha coleccionado entre las regiones de Arica y Parinacota y de los Lagos, y en la isla Masatierra (Robinson Crusoe) en Chile insular. Por el tipo de ambiente donde crece, generalmente no agrícola y menos alterado que el de las especies que claramente se comportan como malezas agrícolas o plantas ruderales tales como Polygonum lapathifolium o P. persicaria, la consideramos como nativa, pese a que Rodríguez et al. (2018) la incluyeron entre las alóctonas asilvestradas.

Materiales estudiados: CHILE. Región de Arica y Parinacota, prov. Arica, río San José, quebrada junto a la playa, $10 \mathrm{~m}$ s.n.m, 14-V-1979, C. Villagrán, M. Kalin, J. Armesto \& P. Uslar, 1012 (CONC). Valle de Azapa, 690 m s.n.m., 22-III-1987, O. Matthei \& R. Rodríguez 381 (CONC). Región de Atacama, prov. de Copiapó, vegas de Copiapó, R.A. Philippi s/n (SGO 48047). Fundo Toledo, $15 \mathrm{~km}$ al norte de Copiapó, $285 \mathrm{~m}$ s.n.m., 25-III-1987, O. Matthei \& R. Rodríguez 443 (CONC). Prov. Huasco, Camino de Vallenar a Freirina, Ilanos de Ferrera, 350 m s.n.m., 13-III-1987, O. Matthei \& R. Rodríguez 143 (CONC). Región de Coquimbo, Prov. Elqui, La Serena, IX1947, B. Sparre 2763 (SGO). Prov. Limarí, tranque Recoleta, 5-12-1930, R. Cabrera 77 (SQF). Rio seco de Los Choros, mina de Los Morros, XI-1971, I. Pizarro de Urízar (SGO 80518). El Palqui, Ovalle, sector tranque La Paloma, 29-III1986, E. Cortés s/n (SQF 16532). prov. Choapa, Chillepín, 
600 m s.n.m., 29-III-1986, M. Le Feuvre s/n (SQF 16571). Prov. Valparaíso, ad ostium fluvium Aconcagua, I-1884, E. Moore s/n (SGO 48037). Quintero, Los Arrayanes, XI-1953, H. Gunckel (SQF 10163). Placilla, 1-01-1961, L.E. Navas 2665 (SQF). Viña del Mar, 2-I-1954, M. Arriagada s/n (SQF 6873). Tunquén, estero Casablanca, 10-50 m s.n.m., 1-XI-2001, S. Teillier 5850 (CONC). Archipiélago Juan Fernández, isla Masatierra, San Juan Bautista, small ravine between Punta La Turbia down toward the sea, 7-II-1990, M. Rondanelli \& A.M. Humaña 11503 (CONC). Prov. Marga Marga, Quilpué, Paso Hondo, 27-10-1985, J. Valdivieso s/n (VALP 1289). Quilpué, paso Hondo, 26-X-1985, S. Díaz, s/n (VALP 951). Prov. Los Andes, río Aconcagua, 12-XI-1954, M. Arriagada $\mathrm{s} / \mathrm{n}$ (SQF 8346). Prov. San Felipe, Panamericana $5 \mathrm{~km}$ E del túnel La Calavera, 7-I-1986, R. Peña s/n (SQF 16451). Prov. San Antonio, El Tabo, quebrada de Córdova, II-1980, I. Meza \& C. Villagrán 875 (SGO). Las Cruces, laguna El Peral, 16-I1985, M.P. Sánchez s/n (SQF 16520). Región Metropolitana, prov. Chacabuco, Batuco, XII-1953, H. Gunckel s/n (SQF
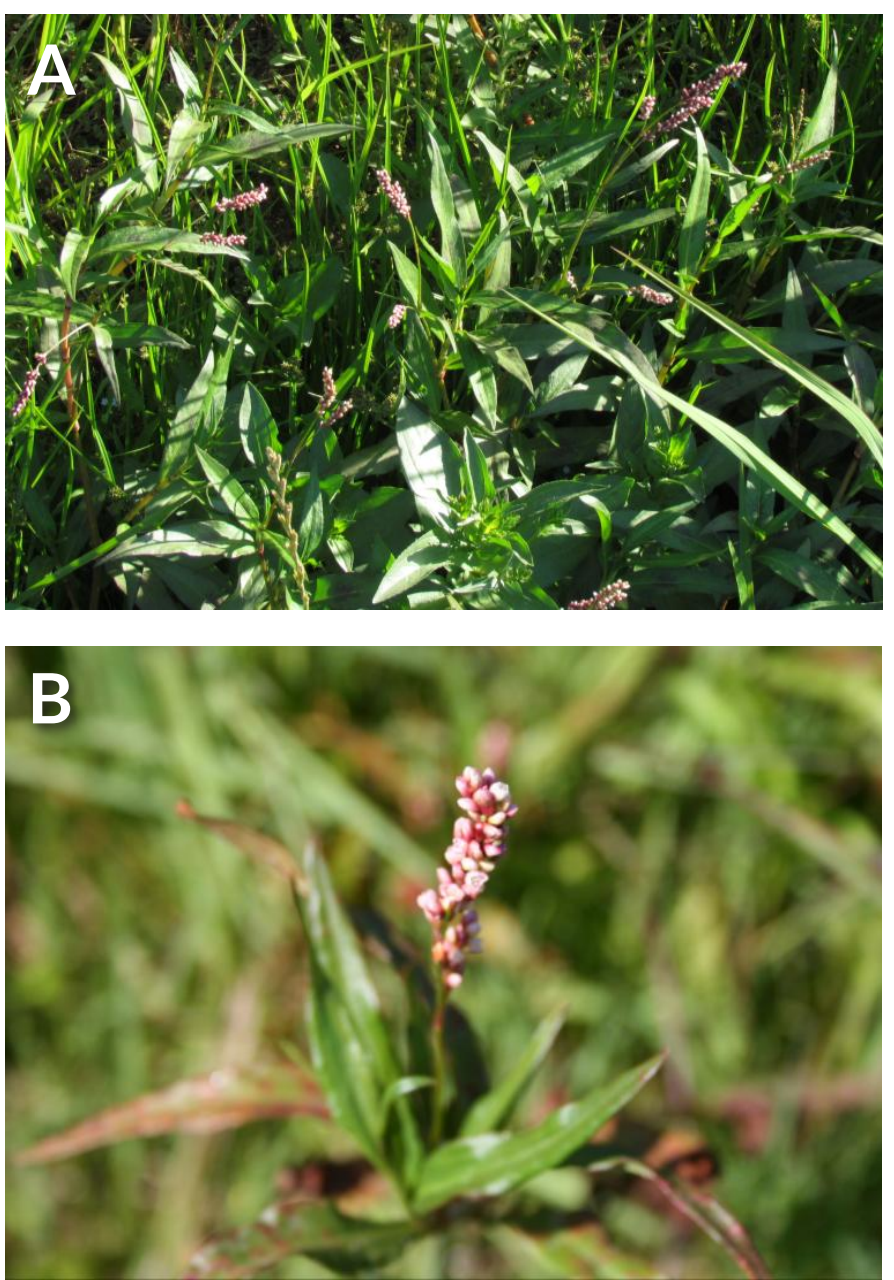

FIgURA 7. P. hydropiperoides Michx. A) Hábito, B) Inflorescencia. / A) Habit, B) Inflorescence. Fotografías: S. Teillier.
10620). Prov. Santiago, Chuchunco, XII-1889, R.A. Philippi s/n (SGO 48036). Quebrada La Plata, Maipú, 12-IV-1988, R. Peña, 406 (SQF). La Cisterna, XI-1953, A. Moreno s/n (SQF 12265). Quebrada de Macul, 4-05-1985, F. Kendall s/n (SQF 16804). Quebrada de Macul, 13-04-1985, L. Núñez s/n (SQF 16801). Cerro San Cristóbal, 18-04-1954, L. E. Navas 598 (CONC, SQF). Pudahuel, IV-1970, M.G. Colombo, S. Hein \& L. Videla s/n (SQF 14383). Peñalolén, 27-12-1954, M. Ayala s/n (SQF 8458), Peñalolén, 600 m s.n.m., X-1942, V. Barahona 286 (SQF). Prov. Cordillera, cajón del Maipo, El Manzano, 14-V-1985, C. Durán s/n (SQF 16500). Cajón del Maipo, km 30, III-1986, C. Grau s/n (SQF 16517). Cajón del Maipo, 2-IV-1987, A. Barrera s/n (SQF 16851). Pirque, 29III-1987, S. Suárez s/n (SQF 16841). Prov. Maipo, hacienda Mansel, III-1888, R.A. Philippi s/n (SGO 48040). Hacienda Mansel, XII-1886, R.A. Philippi s/n (SGO 48041). Región de O'Higgins, prov. Cachapoal, San Vicente de Tagua Tagua, Requegua, 28-III-1986, E. Peralta s/n (SQF 16512). Requinoa, fundo Totihue, II-1652, J. Petit s/n (SQF 4405). Graneros, 1504-1984, R. Zamorano s/n (SQF 16093). Prov. Colchagua, La Palmilla, camino de Paniahue a Peralillo, 160 m s.n.m., 29-XI1989, O. Matthei \& M. Quezada 728 (CONC). Prov. Cardenal Caro, La Estrella, 29-III-1986, A. Campos s/n (SQF 16506). Región de Maule, prov. Curicó, Teno, 500-600 m s.n.m., 19IV-1986, A. Bórquez s/n (SQF 16508). La Huerta, camino de Rauco a Hualañe, 75 m s.n.m., 29-XI-1990, O. Matthei \& M. Quezada 1229 (CONC, SQF). prov. Talca, Constitución, XI1891, K. Reiche s/n (SGO 61783). Bajos de Vilches, I-1960, R. Acevedo (SGO 126736). Talca, IV-1987, M. Reyes 9 (SQF). Fundo Peumo Negro, camino de Talca a El Diamante, 22-I1991, O. Matthei \& M. Quezada 1445 (CONC). Prov. Linares, termas de Quinamávida, 26-I-1986, Maturana \& León s/n (SQF, 16518). Quella sur, 120 m s.n.m., 21-I-1991, O. Matthei \& M. Quezada 1422 (CONC). Región de Ñuble, prov. Diguillín, Chillán, 4 -III-1862, R.A. Philippi s/n (SGO 38691). Chillán, escuela de Agronomía,11-I-1960, O. Matthei s/n (CONC). Prov. Punilla, Niquén, 6-II-1959, O. Matthei s/n (CONC 26983). Región del Biobío, Prov. Concepción, Hualpén, 13XII-1950, M. Ricardi 655 (CONC). Hualpén, Estación de Ecología Terrestre de Hualpén (Laguna Verde), 16-III-2016, N. Viveros, 5004 (CONC). Laguna Lo Galindo, 25 m s.n.m., 30III-1990, P. López 692 (CONC). Laguna Pineda, 50 m s.n.m., 14-XI-1992, N. Mazzeo, M. Rondanelli \& R. Rodríguez 23-E (CONC). Prov. Arauco, Arauco, 1925, F.W. Pennell 12970 (SGO). Contulmo, II-1913, F. Johow s/n (CONC 134012). Contulmo, 10-XII-1918, K. Behn, s/n (CONC 23324). Región de La Araucanía, prov. Malleco, Traiguén, 8-II-1909, A. Koch, (SQF 16799). Región de los Ríos, prov. Valdivia, in paludosis frequens, IV-1834, C. Gay 2035 (SGO). San Juan, I-1881, R.A. Philippi s/n (SGO 48046). San Juan, I-1885, s/n (SGO 48042). 
Corral, Niebla, 11-I-1964, Gunckel 41829 (CONC). Región de los Lagos, Prov. Chiloé, Castro, cordillera de San Pedro, 6-II1982, C. Villagrán \& I. Meza 3838 (SGO).

Observaciones: en el protólogo de Polygonum virgatum se citan dos colecciones; una realizada en "Talcaguano" (Chile) por L.K.A. von Chamisso, y otra, en Brasil por F. Sellow. El material de F. Sellow d. 1559 en Berlín (B cb 100248289) se ha designado como lectotipo y el de HAL (cb 0053998) como isolectotipo, ello, por encontrarse el ejemplar más completo; el ejemplar de L.K.A. von Chamisso, coleccionado en Chile y depositado actualmente en el herbario de Berlín (B 10 0248288) queda como un sintipo.

Navas (1976) mantuvo por separado a Polygonum virgatum y a $P$. persicarioides, no figurando en su tratamiento $P$. hydropiperoides. Luego de examinar numerosos ejemplares de herbario en SGO, CONC y SQF, y observar las plantas en su hábitat se llegó a la decisión de reunir a ambas especies bajo Polygonum hydropiperoides ya que los caracteres que se utilizan para separarlas, al menos en Chile (ancho de la inflorescencia y la pilosidad de las ócreas y de las ocréolas), tienen claramente una variación continua en su morfología.

Existen algunos ejemplares con inflorescencias estrechas, de menos de $4 \mathrm{~mm}$ de ancho, flores más o menos alternadas en el eje, perigonio rosado, con ocréolas y ócreas ciliadas que son morfológicamente similares a Polygonum setosum Bald. ex Ell., pero al menos respecto de los caracteres citados por Cialdella (1989) "cilios más largos que las ocreas y que las ocréolas", estos no se observan en el material chileno; por otro lado, cilios cortos en las ócreas y en las ocréolas también se observan en el material tipo de $P$. hydropiperoides. Una lista de exsiccata con estas características se muestra a continuación:

CHILE. Región de Valparaíso, prov. Los Andes, Los Andes, II1952, E. Silva s/n (SQF 4459). Archipiélago de Juan Fernández, Masatierra, calle La Pólvora, 11-02-1980, C. Marticorena \& E. Ugarte 9202 (CONC). San Juan Bautista, up sidewalk toward El Castillo, 7-II-1990, T.F. Stuessy \& M. Baeza 11476 (CONC). Región Metropolitana, prov. Cordillera, La Obra, 4-I-1954, M. Arriagada s/n (SQF 8988). San Alfonso, 11-I-1954, M. Arriagada s/n (SQF 9070). El Canelo, 29-III-1953, L.E. Navas 952 (SQF). El Laurel, camino El Principal a río Clarillo, 700 m s.n.m., 6-I-1990, E. Bobadilla s/n (CONC 108510). Prov. Santiago, Peñalolén, 27-XII-1954, M. Arriagada 268 (CONC, SQF). Región de O'Higgins, prov. Cachapoal, Graneros, 11-I1986, E. González s/n (SQF 22558). Región de Ñuble, prov. Diguillín, Quillón, laguna Avendaño, 50 m s.n.m., 20-III-1993, N. Mazzeo \& A. Marticorena 30 (CONC). Región de los Lagos, prov. Llanquihue, Puerto Montt, II-1952, O. Ampuero s/n (SQF 4434).

8. Polygonum lapathifolium L., Sp. PI. 1: 360. 1753. Persicaria lapathifolia (L.) Delarbre, FI. Auvergne (ed. 2): 519. 1800. TIPO: Herb. Clifford: 42, persicaria 2 (BM-000557852 Fotografía!) (lectotipo designado por Timson, Watsonia 5: 394. 1963). Polygonum persicaria L. var. vernicosum Cham, et Schldl., Linnaea 3: 43. 1828. TIPO: Brasil, "Brasilia meridionalis", F. Sellow 947 (B cb 101067609 Fotografía!).

Polygonum utriculatum J. Remy, in Gay, Fl. Chile 5: 267. 1849. TIPO: Chile, "in segetis San Carlos, Chiloé, Februario 1836" C. Gay 2034, (holotipo, P cb 00734487 Fotografía! isotipos, SGO 48017!, SGO 48016!). Polygonum valdivianum Phil., Anales Univ. Chile, 91: 488. 1895. TIPO: Chile, "in provincia Valdivia non rarum", R.A. Philippi s/n (lectotipo, SGO 48008! aquí designado, isolectotipo, SGO 38694). Polygonum araucanum Phil., Anales Univ. Chile 91: 489. 1895. TIPO: Chile, "ex Araucania attulit orn.", Februario 1888, W. Calvert, (lectotipo, 48018 SGO! aquí designado, isolectotipo, SGO 38705!). Polygonum glareosum Phil., Anales Univ. Chile. 91: 490. 1895. TIPO: Chile, "in glareosis rivuli de Aculeo dicti provinciae O'Higgins frequens reperitu", R.A. Philippi $s / n$ (lectotipo SGO 48033! aquí designado, isolectotipos, SGO 48032!, SGO 38703!).

\section{Nombre común: Duraznillo}

Hierba anual, de hasta $120 \mathrm{~cm}$ de alto, tallos erectos, estriados, generalmente rojizos, con cilios cortos en los extremos superiores. Hojas cortamente pecioladas, las mayores con cilios cortos en márgenes y nervaduras, las menores, glandulosas o lanosas en el envés, lámina lanceolada, de $5 \times 1 \mathrm{~cm}$, margen entero; ócreas turbinadas, acuminadas, uninervias, glabras, de 3-6 $\mathrm{mm}$ de largo. Flores traslapadas dispuestas en tirsos espiciformes, densos, las brácteas reducidas a sus ócreas que son brevemente acuminadas. Perigonio con 4 tépalos blancos o rosados; estambres 6 , anteras rosadas o amarillas; gineceo con el ovario con 2 estilos, de 0,8 mm de largo. Nuez lenticular muy raras veces trígona, lisa, parda, caras cóncavas, de $2 \mathrm{~mm}$ de largo (Fig. 8).

Distribución geográfica: especie euroasiática; en Chile, alóctona asilvestrada. Planta higrófila, habita en sitios húmedos e inundados. Crece entre las regiones de Valparaíso y de los Lagos; se la ha citado para Juan Fernández (Danton \& Perrier 2006, Rodríguez et al. 2018), pero los ejemplares en CONC pertenecen a $P$. hydropiperoides. 


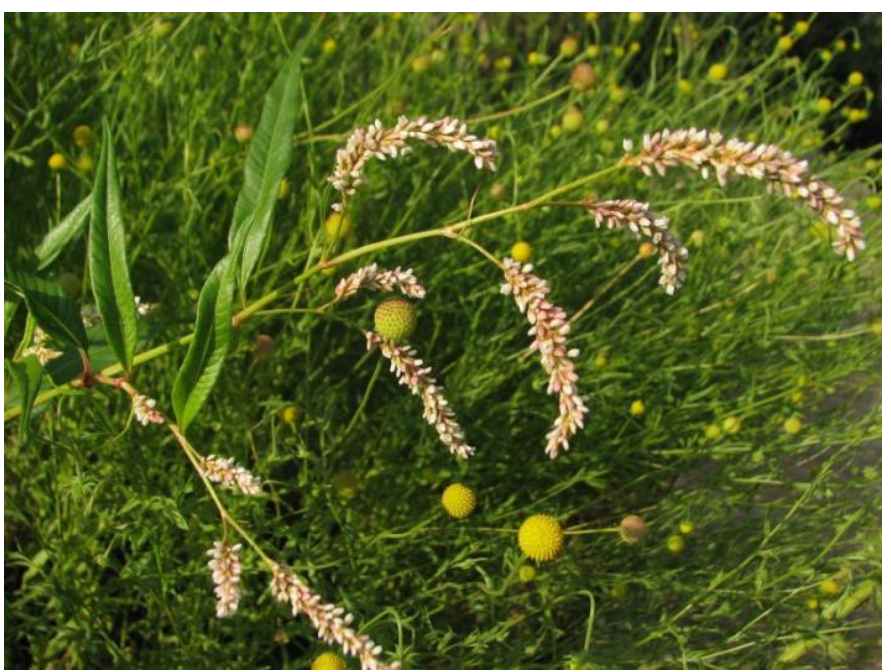

FIgURA 8. Polygonum lapathifolium L. Hábito e inflorescencia. / Habit and inflorescence. Fotografía: C. Ormazábal.

Observaciones: se distingue de las especies anuales afines por sus tirsos espiciformes, densos, con las flores traslapadas y por sus ócreas glabras y sin cilios en el ápice. Polygonum persicaria L., la más cercana, se distingue de P. lapathifolium por la ausencia de glándulas en los pedicelos, en los pedúnculos, en el perigonio y en el envés de las hojas (R. Peña et al. Ms). En ausencia de la parte subterránea puede confundirse con Polygonum hydropiperoides var. hydropiperoides, de la que difiere principalmente por tener las ocréolas acuminadas.

A partir de la observación de los materiales tipo de Polygonum araucanum se propone su sinonimización con $P$. lapathifolium, considerando las ócreas de las brácteas claramente acuminadas y el envés glanduloso de las hojas. Dada la existencia de sintipos para este nombre, se ha lectotipificado con el ejemplar de Calvert $\mathrm{s} / \mathrm{h}$. correspondiente a SGO 48018. Se lo ha elegido por estar más completo y en mejores condiciones.

Polygonum glareosum representa una forma enana de $P$. lapathifolium, con hojas tomentosas en el envés. Es muy similar, además, al ejemplar tipo de Polygonum utriculatum. Se lo ha lectotipificado con el ejemplar de R.A. Philippi $\mathrm{s} / \mathrm{n}$, consignado como SGO 48033 por presentar un mayor número de ejemplares en la muestra.

Polygonum persicaria var. vernicosum fue citado por Cialdella (1989) entre los sinónimos de esta especie. Su referencia es un ejemplar coleccionado en Talcahuano por A. Chamisso.

Polygonum utriculatum concuerda con el tipo de P. lapathifolium por la pilosidad de las hojas, por lo que se conservan los criterios tanto de Small (1892) como de Cialdella (1989).

Polygonum valdivianum, según Small (1892) sería sinónimo de P. persicaria; sin embargo, la morfología del tipo, particularmente la observación de sus ócreas glabras, sin cilios en el ápice, coincide mejor con la de P. lapathifolium. Se lo ha lectotipificado por existir más de un ejemplar tipo con el ejemplar de R.A. Philippi, consignado como SGO 48008 por constituir una muestra más completa y más abundante.

Materiales estudiados: CHILE. Región de Valparaíso, prov. Valparaíso, Viña del Mar, orillas del estero [Marga Marga] 2-I1954, M. Arriagada s/n (CONC 133946, SQF 6874). Quintero, bosque de los arrayanes, 5-XI-1952, H. Gunckel 40535 (CONC). Prov. Quillota, Quillota, I-1884, E. Moore s/n (SGO 48019). Región Metropolitana, prov. Cordillera. El Canelo, 29III-1953, L.E. Navas 952 (SQF). El Manzano, cajón del Maipo, 04-1986, I. Vera s/n (SQF 16505 y 16524). El Volcán, 12-XI1939, Burkart 9350 (SI). Prov. Santiago, Santiago, 1829, C. Gay s/n (SGO 48009). Ad loco fluminis Mapocho, II-1882, F. Philippi s/n (SGO 38712). Cerro San Cristóbal (lado SE), 7-XII-1953, L.E. Navas 305 (SQF). Providencia, 14-I-1954, M. Arriagada s/n (SQF 9640). Río Mapocho, 20-III-1921, Hicken 35 (SI). Rinconada de Lo Cerda, Internado, 840 m s.n.m., 19-II1956, F. Schlegel 1031 (CONC). Prov. Maipo, estero Champa, 11-XI-1986, 50 m s.n.m., F. Aguayo s/n (SQF 16684). Región de O'Higgins, prov. Cachapoal, San Vicente de Tagua Tagua, 15-04-1986, M. Vergara s/n (SQF 16504). Región de Maule, prov. Linares, Longaví, La Granja, 140 m s.n.m., 14-III-1988, R. Rodríguez \& M. Baeza 2286 (CONC). Región de Ñuble, Los Lleuques, 25-04-1954, M. Ledezma 172 (SQF). General Cruz, fundo San Miguel, 10-II-1959, O. Matthei s/n (CONC 26926). Región de Biobío, fundo Santa Olga, II-1954, E. Gautier s/n (CONC 133953). Prov. Concepción, ad portum Talcaguano, Chile, Von Chamisso s/n (B como P. persicaria var. vernicosum). Hualpén, 17-XI-1925, E. Barros V. 1092 (CONC). Fundo Trinitarias, entre Concepción y Florida, 29-XII-1934, A. Pfister s/n (CONC 1214). Hualqui, Pichaco, 13-I-1937, C. Junge $\mathrm{s} / \mathrm{n}$ (CONC 5933). Talcahuano, isla Rocuant, $5 \mathrm{~m}$ s.n.m., 10-I-2005, R. Rodríguez, P. Claret \& C. Sanhueza 4064 (CONC). Prov. Arauco, isla Mocha, laguna Hermosa, E. Weldt \& R. Rodríguez 1153/448 (CONC). Región de la Araucanía, prov. Malleco, Mininco, 7-XII-1952, H. Schwabe (CONC 13689). Prov. Cautín, "Cautín", 1914, V. Baeza (CONC 133963). Fundo Quintrilpe al norte de Vilcún, 350 m s.n.m., 8-I-1991, O. Matthei \& M. Quezada 1278-B (CONC, SQF). Región de los Ríos, prov. Valdivia: San Juan, II-1859, R. A. Philippi s/n (SGO 48011). Valdivia, II-1957, G. Kunkel 44 (CONC). Prov. Ranco, Río Bueno, 26-27-II-1948, B. Sparre 
4614 (SGO). Región de los Lagos, prov. Osorno, Pichapicha, entrada a Quilacahuín 45 m s.n.m., 19-III-1990, O. Matthei \& M. Quezada 1027 (CONC). Prov. Chiloé, Piruquina, 7-III1932, C. Junge s/n (CONC 2273).

9. Polygonum maritimum L., Sp. Pl. 1: 361. 1753. TIPO: "Habitat Monspelii, in Italia, Virginia" (lectotipo: Löfling S.N., Herb. Linn. No. 510.22 LINN Fotografía!), designado por Styles, Watsonia 5: 212. 1962.

Polygonum chilense K. Koch, Linnaea 22: 206. 1849. Polygonum maritimum var. chilense (K. Koch) Skottsb., Kgl. Sv. Vet. Akad. Handl. 50 (3): 25. 1913. TIPO: "Chile", B. von Besser s/n (holotipo, B cb 100279388 Fotografía!).

Polygonum sanguinaria J. Remy in Gay, Fl. Chil. 5: 270. 1851. TIPO: "Chile, Coquimbo, Tige très longue trainant sur le sol sablonneux du bord de la mer. Fleurit en octobre, commun", $C$. Gay 35 (lectotipo P cb 00734478 Fotografía! aquí designado; isolectotipos, P cb 00734479 Fotografía!, P cb 00734480 Fotografía!).

Nombre común: Sanguinaria

Hierba perenne. Tallos leñosos, postrados, pardo-rojizos, estriados, de 10-60 cm de largo, ramas áfilas en la parte inferior, en algunos ejemplares casi completamente cubiertas por las ócreas. Hojas color verde-cenicientas, subsésiles, coriáceas o algo carnosas, con 8-12 nervios prominentes, casi paralelos, elíptico-lanceoladas, de 5-30 x 1-6 mm, margen revoluto; ócreas blancas, laciniadas, de $8-20 \mathrm{~mm}$ de longitud, más largas que la mitad del entrenudo hasta cubrirlo completo en la parte superior; brácteas reducidas a las ócreas. Inflorescencia en cimas (cincinos) axilares, con 1-7 flores. Perigonio con 5 tépalos, blancos con la base rosada; estambres 8, anteras amarillas; ovario de $1 \mathrm{~mm}$ de largo, estilos 2 o 3, estigmas capitados. Nuez ovoide, trígona en el ápice o piramidal, brillante, de color pardo-anaranjado (Fig. 9).

Distribución geográfica: especie subcosmopolita, nativa en Chile. Se la ha coleccionado desde la Región de Coquimbo hasta la de Magallanes. Especie litoral, crece en las dunas consolidadas y en las rocas.

Observaciones: Polygonum maritimum presenta variaciones morfológicas aparentemente ligadas con la estacionalidad. Los renuevos primaverales tienen los entrenudos casi totalmente cubiertos por las ócreas y las hojas son más estrechas; a medida que avanza la estación, los entrenudos de las ramas se alargan y las ócreas foliares apenas los alcanzan o son más cortas y las hojas, más anchas. Es posible también que la variación que se ha observado esté relacionada con los diferentes tipos de suelo donde medra, sea arenoso, rocoso o salino.

Skottsberg (1913) y más tarde Moore (1983) consideraron a Polygonum chilensis como una variedad de $P$. maritimum, pero siguiendo a Crespo \& Pérez Moreau (1984) y a Cialdella (1989) se la ha incluido aquí entre los sinónimos de P. maritimum.

Respecto de Polygonum sanguinaria, descrita por Rémy para el litoral de Coquimbo, luego de revisar el material tipo y ejemplares de herbario, se ha establecido que no existen caracteres que justifiquen mantenerla como una entidad diferente a $P$. maritimum. Se trata de dos hierbas perennes, con hábito, distribución geográfica y hábitat muy coincidentes. Este criterio difiere del de los catálogos de Marticorena \& Quezada (1985), Zuloaga et al. (2008) y Rodríguez et al. (2018). Se ha designado un lectotipo para Polygonum sanguinaria por existir en $\mathrm{P}$ tres ejemplares coleccionados por C. Gay con el mismo número de colector, eligiéndose el más completo.

Polygonum stypticum fue mencionada para Chile por Cialdella (1989), pero su presencia es dudosa y quizás refiera a ejemplares de $P$. maritimum, dos entidades algo similares en su morfología, pero los ejemplares de P. maritimum son mucho menos robustos.

Materiales estudiados: CHILE. Región de Coquimbo, prov. Elqui, Serena, IX-1836, C. Gay 2036 (SGO). 40 km al sur de La Serena, 15-IX-1957, M. Ricardi \& C. Marticorena 4315/700 (CONC). Prov. Limarí, parque nacional Fray Jorge, 28-III-1947, C. Jiles 48 (SQF). Tongoy, II-1953, E. Gautier s/n

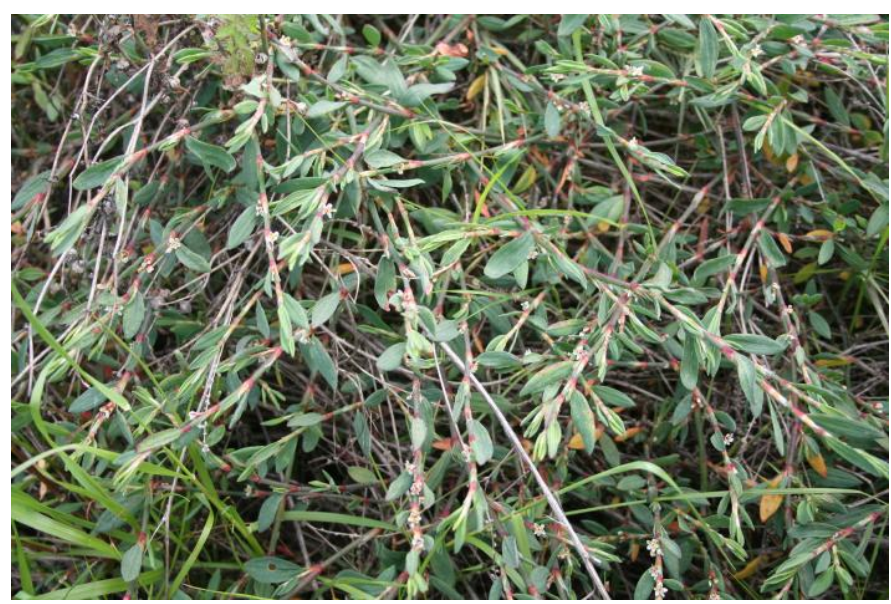

FIGURA 9. Polygonum maritimum L. Ramillas tendidas y hojas. / Prostrate branches and leaves. Fotografía: S. Teillier. 
(SQF 4460). Prov. Choapa, Los Vilos, 8-I-1976, B. Holton 2 (SGO). Región de Valparaíso, prov. Petorca, Los Molles, 1809-2014, S. Teillier, J. Delaunoy \& R. Lund 8613 (CONC). Prov. Valparaíso, Quintero, 5 m s.n.m., 17-X-1951, J. Ibáñez s/n (SQF 3215). Quintero, XI-1952, L.E. Navas s/n (SQF 14390). Quintero, XI-1953, H. Gunckel s/n (SQF 10398). Quintero, Loncura, XII-1953, H. Gunckel 27373 (CONC, SQF). Mantagua, Quintero, 13-III-1976, J. Saravia s/n (SQF 15391). Quintay, 29-III-1986 P. Bize s/n (SQF 16541). Prov. San Antonio, Algarrobo, El Canelillo, 24-II-1955, E. Kausel 4142 (SGO). Cartagena, 3-XI-1928, R. Cabrera (SQF 14393), Santo Domingo, 28-I-1960, A Rex s/n (SQF 16798). Rocas de Santo Domingo, 6-I-1976, B. Holton 18 (SGO). Región de O'Higgins, prov. Cardenal Caro, Navidad, La Boca, 50-100 m s.n.m, 13-10-1973, G. L. Stebbins, L. Robres 8602 (SGO). Pichilemu, fundo Millaco, II-1954, M. Arriagada s/n (SQF 5508). Región del Maule, Prov. Curicó, desembocadura de la laguna de Llico, II-1969, C. Villagrán \& G. Tapia (SGO 78983). Prov. Talca, Constitución, II-1895, K. Reiche s/n (SGO 48056). Región de Ñuble, prov. Itata, Cobquecura, 13-IV-1954, M. Ledezma 203 (SQF). Región del Biobío, prov. Concepción, isla Mocha, II-1902, K. Reiche, s/n (SGO 48057). Dichato, 25-XI1943, R. Silva 318 (SGO). Tomé, Cocholgüe, cerca del mar, 4-I-1935, A. Pfister s/n (CONC 5278). Talcahuano, península Tumbes, 25-XII-1935, C. Junge s/n (CONC 5638). Lirquén, 11-XII-1950, M. Ricardi s/n (CONC 11017). Hualpén, playa Blanca, 26-XII-1941, A. Pfister s/n (CONC 23318). Prov. Arauco, Arauco, 1925, F.W. Penell 12910 (SGO). Región de la Araucanía, prov. Cautín, Puerto Saavedra, 3-II-1963, L.E. Navas 3147 (SQF). Región de los Ríos, prov. Valdivia, Mehuín, 15-I-1959, G. Montero 6155 (CONC). Corral, 2-II1932. H. Gunckel 3126 (SQF). Prov. Ranco, Hueicolla, XI1867, R.A. Philippi, s/n (SGO 38697). Hueicolla, 10-II-1988, M.F. Gardner \& S.G. Knees 4140 (CONC). Trumao, barra del rio Bueno, 50 m s.n.m., V-1933, A. Hollermayer 618 (SGO). Región de los Lagos, prov. Osorno, Pucatrihue, 11-12-I-1948, B. Sparre 3871 (SGO). Prov. Chiloé, Chepu, 15-X-1958, E. J. Godley 222 (SGO). Ancud, 12580, F.W. Penell 12910 (SGO). Islas Desertores, Talcán, 25-29-I-1982, C. Villagrán 3436 (SGO). Chiloé National Park, $30 \mathrm{~km}$ north of Cucao, at the mouth of the río Anay, 27-I-1988, M.F. Gardner 3589 (CONC). Región de Magallanes, prov. Magallanes, route 225 east at $120 \mathrm{~km}$ post from Punta Arenas, just past intersection with Río San Gregorio, 9-X-1971, T. R. Dudley, R.N. P. Goodall \& G. Crow 97 (SGO). Prov. Tierra del Fuego: Bahía Inútil, estancia Cameron, 19-III-1964. David Moore, 1075. (SGO). Sector Río Cóndor, Forestal Trillium, delta del río, orilla sur, 5-II-1995, E. Pisano, Henríquez \& E. Domínguez 7934 (CONC). Isla Grande de Tierra del Fuego, desembocadura del río Azopardo, 30-I2012, S. Pfanzelt 690 (CONC).
10. Polygonum orientale L. Sp. Pl: 362. 1753. Persicaria orientalis (L.) Spach, Hist. Nat. Vég. Phanérogames 10: 537. 1841. Amblygonum orientale (L.) Nakai ex T. Mori, Enum. PI. Corea 129. 1922. TIPO: "Habitat en Oriente, India" Lectotipo. Herb. LINN. No.510.16 (LINN Fotografía!), designado por Wijnands, Bot. Commelins: 175. 1983.

Hierba anual, tallos densamente pilosos, de hasta de 0,5-2 m de altura, más o menos fistulosos, ascendentes, medianamente ramificados. Hojas pecioladas, escasamente pilosas; aovadas a cordadas, de (3) $8,5-20 \times(1,5) 3,5-10 \mathrm{~cm}$, acuminadas hasta aristado-acuminadas, margen ciliado; ócreas de 15-20 mm, fimbriadas, verdes a pardo claras; brácteas reducidas a sus ócreas, éstas turbinadas y ciliadas. Inflorescencia un tirso espiciforme, oblongo, de 4-9 cm de largo; cimas (cincinos) con 6 a 11 flores; pedicelos de 4-6 mm. Perigonio de 5 tépalos, de 4-5 mm, color rosado a rosado oscuro, raras veces blanco; androceo con 6-7 estambres, anteras amarillas. Ovario con 2 estilos. Nuez negruzca, lenticular, biconvexa, brillante (Fig. 10).

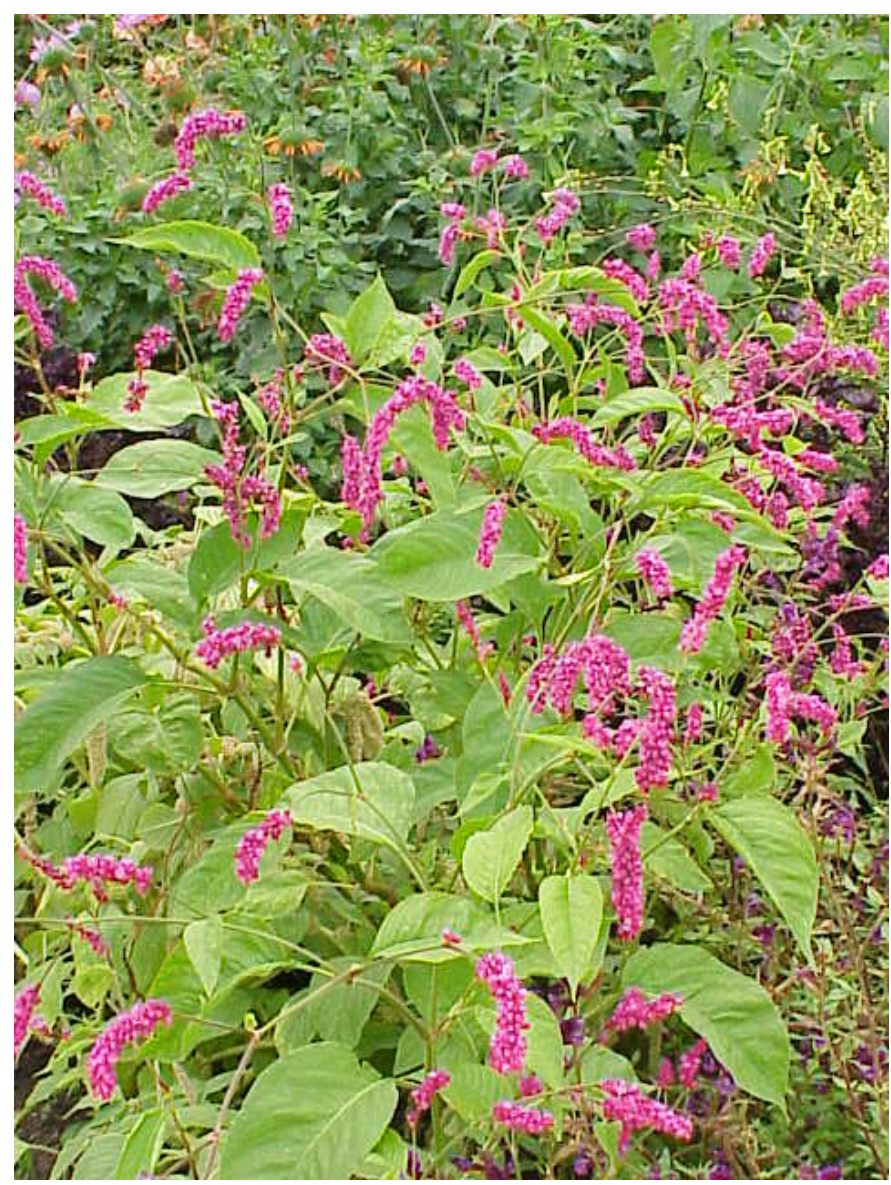

Figura 10. Polygonum orientale L. Ramas e inflorescencias. / Branches and inflorescences. Imagen de referencia tomada del sitio https://www. commons.wikipedia.org, Autor: Kurt Stueber. 
Distribución geográfica: especie de origen asiático. En Chile, es alóctona asilvestrada. Crece en las regiones Metropolitana, del Biobío y de la Araucanía (Rodríguez et al. 2018).

Observación: planta ornamental; algunas veces se escapa del cultivo.

Material estudiado: CHILE. Región de la Araucanía, prov. Cautín, Pucón, II-1935, A. Pfister (CONC 1981). Fundo El Colorado, arenales del río Claro, 21-II-1952, A. Garaventa 4958 (CONC).

11. Polygonum persicaria L., Sp. PI. 1: 361. 1753. Persicaria maculosa Gray, Nat. Arr. Brit. PI. 2: 269. 1822. TIPO: "Habitat in Europae cultis" Lectotipo. Herb. Burser Iv: 101, espécimen de la izquierda (UPS), designado por Ekman \& Knutsson, Nordic J. Bot. 14: 24. 1994.

Nombre común: Duraznillo

Hierba anual, tallo erecto o postrado-ascendente, de hasta $80 \mathrm{~cm}$, ramificado. Hojas casi sésiles, glabras o algo híspidas (estrigosas) en el envés, principalmente sobre la nervadura, lanceoladas, de 6-15 x 1-3,5 cm, con frecuencia con una mancha central violáceo oscura en el centro; ócreas membranosas, verdosas o apenas manchadas de rojo, de 6-10 mm, ciliadas y pilosas (estrigosas) en las nervaduras. Inflorescencia, un tirso espiciforme, denso, ininterrumpido de $10-45$ x 7-10 (12) mm. Perigonio de 2-3 mm de largo, tépalos, 5 , de color rosado intenso o pálido; androceo con 6-8 estambres; gineceo con ovario 2-3 carpelar, estilo bífido. Nuez de 2-3 mm, lenticular o raras veces trígona, de color negro brillante (Fig. 11).

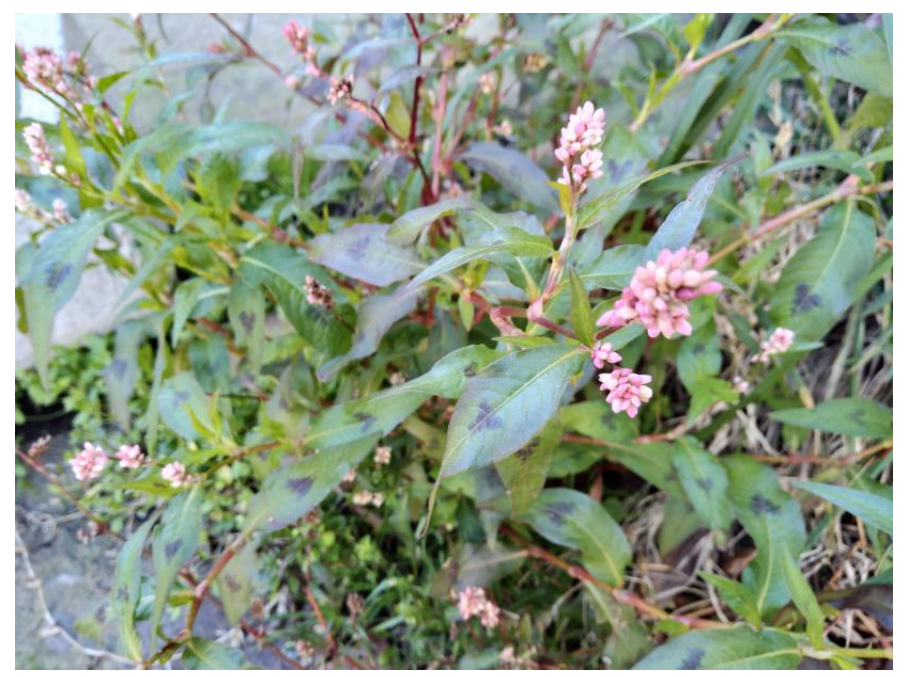

Figura 11. Polygonum persicaria L. Hojas e inflorescencias. / Leaves and inflorescences. Fotografía: S. Teillier.
Distribución geográfica: especie de origen europeo; en Chile, alóctona asilvestrada. Habita desde la Región de Coquimbo hasta la de Magallanes (Rodríguez et al. 2018). Planta higrófila, más frecuente en áreas agrícolas con riego.

Observaciones: especie afín a Polygonum lapathifolium, con la que suele hibridar (Villar 1990). En el catálogo de Rodríguez et al. (2018) figura como Persicaria maculosa Gray. En los herbarios es frecuente encontrar especímenes atribuidos a esta especie que corresponden a Polygonum lapathifolium o a P. hydropiperoides. De Polygonum lapathifolium se diferencia principalmente por las ócreas foliares ciliadas y pestañosas en el margen, siendo mucronadas en P. lapathifolium; de $P$. hydropiperoides se distingue por los rizomas, por sus inflorescencias, generalmente más anchas que $4 \mathrm{~mm}$, o por sus ócreas y por sus ocréolas con frecuencia ciliadas y pestañosas.

Material estudiado: CHILE. Región de Coquimbo, prov. Limarí, estancia Junta de Chingoles, mina de Los Morros, 2000 m s.n.m., XI-1971, I. Pizarro de Urízar s/n (SGO 84661). Región de Valparaíso, prov. San Felipe, Llay-Llay, km 82 Panamericana Norte, 7-IV-1985, I. S. Cajigal s/n (SQF 16803). Prov. Quillota, San Isidro, 95 m s.n.m., 12-XI-1987, O. Matthei \& M. Quezada 372 (CONC). Región Metropolitana, prov. Cordillera, camino a San José de Maipo, 960 m s.n.m., III-1987, M. Foncea s/n (SQF 16850). Cajón del Maipo, 28III-1986, A.M. Martínez s/n (SQF 16587). Lagunillas, 21-III1986, V. González s/n (SQF 16616). Prov. Santiago, La Granja, 21-XII-1986, L. Quiñones s/n (SQF 16718). Maipú, templo votivo, 12-IV-1988, R. Peña 413 (SQF). La Pintana, campus Antumapu, Universidad de Chile, 18-XI-1975, M. Valenzuela $\mathrm{s} / \mathrm{n}$ (SGO). Santuario de la naturaleza Yerba Loca, ladera al SO del estero Yerba Loca, 1700 m s.n.m., 31-I-1999, M.T. KalinArroyo \& A.M. Humaña 99857 (CONC). Región de O'Higgins, prov. Cachapoal, La Compañía, 4-I-1994, R. Peña s/n (SQF 22856). Camino de Coinco a Quillehue, 3-V-1986, V. Soto s/n (SQF 16513). Prov. Colchagua, Las Peñuelas, camino de San Fernando a Nancagua, 28-XI-1989, O. Mattei \& M. Quezada 688 (CONC). Región del Maule, Prov. Curicó, Rauco, parcela El Naranjo, 190 m s.n.m., 23-XI-1989, E. Bobadilla 177 (CONC). Prov. Talca, predio Venecia, $1 \mathrm{~km}$ al sur de Talca, 16-III-1988, R. Rodríguez \& C. Baeza 2343 (CONC). Prov. Linares, 44 km desde Las Ánimas, sector vegas del Molino, 9-III-1999, E. Ruiz \& P. López 1427 (CONC). Región de Ñuble, Prov. Punilla, San Carlos, fundo Las Encinas de Pomuyeto, 3-II-1959, O. Matthei s/n (CONC 26987). Niblinto, fundo El Carmen de Bustamante, 19-I-1959, O. Matthei s/n (CONC 26984). Prov. Diguillín, hacienda Rucamanqui, 15-I-2001, C. Baeza \& R. Rodríguez, 2866 (CONC). Región del Biobío, puente El 
Roble, río Itata, 65 m s.n.m., 19-XI-1992, R. Rodríguez \& A. Marticorena 2907 (CONC). Prov. Biobío, parque nacional Laguna del Laja, 909 m s.n.m., 4-II-2005, A. Marticorena, A. Jiménez \& A. Pauchard, 287 (CONC). Región de la Araucanía, prov. Malleco, Curacautín, 28-II-1939, A. Burkart 9450 (SGO). Fundo La Alianza, camino de Traiguén a Galvarino, 230 m s.n.m., 10-I-1991, O. Matthei \& M. Quezada 1378 (CONC). Prov. Cautín, Temuco, isla río Cautín, 25-XI-1961, G. Montero 6491 (CONC). General López, 210 m s.n.m, N. Espinoza s/n (CONC 101380). Región de los Ríos, Valdivia, XII-1961, H. Gunckel 37673 (CONC). Neltume, reserva biológica Huilo Huilo, pampa Bandurrias, 545 m s.n.m., 22-I-2011, S. Teillier, A. Marticorena, J. Macaya \& C. Bonnemaison 6820 (CONC). Neltume, reserva biológica Huilo Huilo, camino al volcán Choshuenco, 615 m s.n.m., 12-II-2011, S. Teillier, J. Delaunoy \& C. Bonnemaison 7446 (CONC). Región de los Lagos, prov. Osorno, Los Castaños, Río Negro, 02-1986, E. Nahum s/n (SQF 16509). Osorno, Blaña. Pichil-Damas, 17-II-1943, A. Ponce 215 (CONC). Prov. Llanquihue, Puerto Octay, fundo Centinela, 60 m s.n.m., II-1942, E. Pisano \& D. Geni 1141 (SGO). Las Chilcas, camino a Maullín, 20 m s.n.m., 17-III-1990, O. Matthei \& M. Quezada 933 (CONC). Prov. Chiloé, Chacao, 29-III-1984, H. Eisele s/n (SQF 16817). Camino de Ancud a Achao, 19-II-2007, M. Muñoz 4870 (SGO). Isla Grande de Chiloé, Compu, 20 m s.n.m., 16-III-1990, O. Matthei \& M. Quezada 895 (CONC). Palena, Hornopirén, San Ignacio de Huinay, J. L. Fernández, L. Medina, R. Morales, F. Muñoz, JLF 30786 (CONC, SGO).

\section{Especies dudosas o excluidas:}

Polygonum acre Kunth, Nov. Gen. Sp. (quarto ed.) 2: 179. 1817 [1818]. La mayor parte de los materiales etiquetados por R.A. Philippi en SGO corresponden a P. hydropiperoides. Estos materiales al parecer fueron la base de la cita de la especie en el catálogo de F. Philippi (1881).

Polygonum berteroi Phil., Linnaea 29: 38. 1857-58. El ejemplar tipo (SGO 40869), que incluye un espécimen completo, y que coincide con la diagnosis original de R.A. Philippi, contiene dos etiquetas, una de C. Gay: "en arenosis subalpinis ad Cauquenes [baños]", C. Gay 2040; y otra de C. Bertero, con la mención: "P. aviculare caule erecto. In pratis humidis et ad vias prope Rancagua", Bertero s/n, 12-1828. Por ende, si bien se puede identificar a Polygonum berteroi con el ejemplar de SGO, no es posible inferir si este espécimen corresponde a la colección de Gay o a la de Bertero.

Polygonum brasiliense K. Koch, Linnaea 22 (2): 206-207. Citada por Cialdella (1989) para Chile, en las regiones de Aysén y
Magallanes, mención recogida por Zuloaga et al. (2008) y Rodríguez et al. (2018). Si bien actualmente se la considera como un sinónimo de Polygonum aviculare, su presencia en Chile es posible que deba referirse a $P$. delfinii, especie que crece en la península de Taitao [localidad que figura en la etiqueta del material utilizado como cita por Cialdella (1989): C. Muñoz (6194 SGO)].

Polygonum chiloense, nombre en Marticorena \& Quezada (1985) error por Rumex chiloensis Rchb. f.

Polygonumminus Huds., Fl.Angl. 1:148.1762.Superficialmente se trata de plantas muy similares a $P$. hydropiperoides. Small (1892) la propone para Chile, pero no se la ha registrado en los herbarios CONC, SGO, SQF, ni se la ha encontrado en el campo.

Polygonum mite Schrank, Baier. Fl. 1: 668. 1789. Fue citado por San Martín et al. (2001) en un trabajo sobre la flora del estero Reñaca, comuna de Viña del Mar (Región de Valparaíso), pero los ejemplares mencionados no han podido ser localizados. Marticorena \& Quezada (1985) ya había citado esta especie para Chile, situación refrendada por Rodríguez et al. (2018). Dado que, según los datos de GBIF, Polygonum mite no figura en Sudamérica, es posible que por los caracteres morfológicos se trate de algún material correspondiente a $P$. hydropiper o de P. hydropiperoides, dos especies de las que, en ausencia de las partes subterráneas, resulta complejo distinguir.

Polygonum ramosissimum Michaux, Fl. Bor. Amer 1: 237. 1803. Es citada por Navas (1976), pero es una especie que crece en América del Norte y no se encuentra en Chile.

\section{AGRADECIMIENTOS}

Al personal de los herbarios CONC, SGO y SQF por su disposición para consultar aquellos materiales indispensables para esta revisión. A Thomas Rouillard del Museo de Historia Natural de Angers (Francia), por la fotografía del tipo de Polygonum arenastrum. A Fernando O. Zuloaga, del Instituto Darwinion (Argentina), por su ayuda con la nomenclatura. A Ana María Cialdella, también del Instituto Darwinion por ayudarnos en varias consultas sobre el contenido de este trabajo. A los colegas chilenos, María Victoria Legassa y César Ormazábal por sus fotografías. A Alicia Marticorena, curadora del herbario CONC (Chile), por sus aportes para mejorar el manuscrito y a dos editores anónimos que nos ayudaron con sus sugerencias. 


\section{REFERENCIAS}

Choudhary, R.K., Park, S.H., Lee, J. 2012. Phylogeny and systematics of Indian Polygonum sensu lato in the subfamily Polygonoideae based on ITS sequences of nuclear ribosomal DNA. Genetics and Molecular Research 11(4): 4370-4382. Doi: 10.4238/2012.

Cialdella, A.M. 1989. Revisión de las especies argentinas de Polygonum s.l. (Polygonaceae). Darwiniana 29: 179-246.

Costea, M., Tardif F.J. 2004. The biology of Canadian weeds. 131. Polygonum aviculare L. Canadian Journal of Plant Science 85(2): 481-505.

Crespo, S., Pérez Moreau, R. 1984. Polygonaceae. En: Correa, M.N. Flora Patagónica, Colección Científica INTA 8(4a): 60-98.

Danton, P., Perrier, C. 2006. Nouveau catalogue de la flore vasculaire de l'archipel Juan Fernández (Chile). Acta Botanica Gallica 153(4): 399-587.

Fuentes, N., Sánchez, P., Pauchard, A., Urrutia, J., Cavieres, L., Marticorena, A. 2014. Plantas Invasoras del Centro-Sur de Chile: Una Guía de Campo. Laboratorio de Invasiones Biológicas (LIB), Concepción, Chile. http://www.lib.udec. cl/wp-content/uploads/2017/11/plantas-invasoras.pdf

Hong, S.P. 1992. Taxonomy of the genus Aconogonon (Polygonaceae) in Himalaya and adjacent regions. Acta Universitatis Upsaliensis, Symbolae Botanicae Upsalienses 30: 99.

Li, A., Grabovskaya-Borodina, A.E., Hong, S., McNeill, J., Ohba, C, Park, C. 2003. Flora of China. Zhengyi, W., Raven, P.H., Deyuan, H. (eds.), Vol. 5. Ulmaceae through Basellaceae: 277-350. Science Press, Beijing \& Missouri Botanical Garden Press, St. Louis.

Mabberley, D.J. 1987. The plant-book. A portable dictionary of the higher plants. Cambridge University Press, Cambridge. $706 \mathrm{pp}$.

Marticorena, C., Quezada, M. 1985. Catálogo de la flora vascular de Chile. Gayana Botánica 42(1-2): 5-157.

Matthei, O. 1995. Manual de las malezas que crecen en Chile. Alfabeta Impresores, Santiago. 545 pp.

Mertens, T.R., Raven, P.H. 1965. Taxonomy of Polygonum, section Polygonum (Avicularia) in North America. Madroño 18: 85-92.

Moore, D. 1983. Flora of Tierra del Fuego. Anthony Nelson, Shrewsbury, England. 396 pp.

Navas, L.E. 1976. Flora de la cuenca de Santiago. Vol. II. Ediciones de la Universidad de Chile, Santiago, Chile. 559 pp.

Philippi, R.A. 1864. Plantarum novarum chilensium. Centuriae inclusis quibusdam Mendocinis et Patagonicis. Linnaea 33: 1-308.

Philippi, R.A. 1873. Descripción de las plantas nuevas incorporadas últimamente en el herbario chileno. Anales de la Universidad de Chile 41: 663-746.

Philippi, R.A. 1895. Plantas nuevas chilenas de las familias que corresponden al tomo $\mathrm{V}$ de la obra de Gay. Anales de la Universidad de Chile 90: 187-230.

Rémy, J. 1851. Polygonum. En: Gay, C. Historia física y política de Chile, Botánica [Flora Chilena], Vol. 5. Paris, y Museo de Historia Natural de Santiago, Chile.

Rodríguez, R., Marticorena, C., Alarcón, D., Baeza, C.M., Cavieres, L., Finot, V.L., Fuentes, N., Kiessling, A., Mihoc, M., Pauchard, A., Ruiz, E., Sánchez, P., Marticorena, A. 2018. Catálogo de las plantas vasculares de Chile. Gayana Botánica 75(1): 1-430.

Ronse Decraene, L.P., Akeroyd, J.R. 1988. Generic limits in Polygonum and related genera (Polygonaceae) on the basis of floral characters. Botanical Journal of the Linnean Society 98(4): 321-371.

San Martín, C., Ramírez, C., San Martín, J., Villaseñor, R. 2001. Flora y vegetación del estero Reñaca (V Región, Chile). Gayana Botánica 58(1): 31-46.

Schuster T.M., Reveal, J.L., Kron, K.A. 2011. Phylogeny of Polygoneae (Polygonaceae: Polygonoideae). Taxon 60(6): 1653-1666.

Skottsberg, K. 1913. Botanische Ergebnisse. Schwedischen Expedition nach Patagonien und dem Feuerlanden 19071909. III. 3. A botanical survey of the Falkland Islands, Kongliga Svenska vetenskapsakademiens handlingar 50(3): 25.

Small, J.K. 1892. A preliminary list of American species of Polygonum. Bulletin of the Torrey Botanical Club 19(12): 351-370.

Stevens, W.D., Ulloa, C., Pool, A., Montiel, O.M. (Eds.). 2001. Flora de Nicaragua. Vol. 85, tomos I, II y III. Missouri Botanical Garden Press. St. Louis, Missouri.

Styles, B.T. 1962. The taxonomy of Polygonum aviculare and its allies in Britain. Watsonia 5: 177-214.

Thiers, B.M. 2020. The World's Herbaria 2019: A summary report based on data from Index Herbariorum. Issue 3,0 (10 de enero) $20 \mathrm{pp}$.

Villar, L. 1990. Polygonum L. Flora Iberica, II. Castroviejo, S. (coord. gen.). Real Jardín Botánico, CSIC, Madrid.

Webb, D.A., Chatter, A.O. 1964. Polygonum. En: Tutin, T.G., Heywood, N.A., Burges, D.M., Moore, D., Valentine, D.H., Walters, S.M., Webb, D.A. (Eds.) Flora Europaea, Vol 1: 76-80. University Press, Cambridge, UK.

Zuloaga, F.O., Morrone, O., Belgrano, M.J. (Eds.) 2008. Catálogo de las plantas vasculares del Cono Sur. Actualizado 2019: Zuloaga, F.O., Belgrano, M.J., Zanotti, C.A. Darwiniana, nueva serie 7(2): 208-278. Base de datos asociada en: http://www.floraargentina.edu.ar/ Flora del Cono Sur. Accedida: Julio 20, 2020.

Received: 09.06.2020

Accepted: 07.10.2020 